\title{
LECTURE DE ROUSSEAU (*)
}

"La question si cavalièrement décidée dans notre siècle sera mieux discutée dans un autre quand la haine dans laquelle on entretient le public cessera d'être fomentée: et quand dans des générations meilleures celle-ci aura été mise à son prix, ses jugements formeront des préjugés contraires: ce sera une honte d'en avoir été loué, et une gloire d'en avoir été haï".

\section{(J. J. Rousseau)}

\section{Les Métamorphoses de l'Oeuvre}

Le problème de la lecture de Rousseau, des conditions qui la rendent possible et des obstacles que s'y opposent, est donc posé à l'intérieur même de son oeuvre. Le texte en exergue indique le futur comme le seul lieu dans lequel il pourrait être finalement compris comme l'unique point-de-vue auquel l'oeuvre pourrait enfin offrir sa vérité en transparence; il dénonce aussi la mauvaise volonté, la haine comme la puissance qui ferme à ses contemporains tout accès à sa pensée et qui réussit à imposer une image entièrement déformée de sa propre existence. On pourrait y voir la trace d'une théorie de la dépendance de l'entendement par rapport à la volonté, ainsi que de la "viabilité historique des idées", des liens complexes et indirects qui les nouent au Siècle: une éthique

(*) Esste texto, escrito em janeiro e fevereiro de 1970, foi apresentado em março do mesmo ano ao Centre National de la Recherche Scientifique em Paris como plano de trabalho, justificando o pedido de uma bôlsa dessa instituição. Nem um ensaio pois, nem um exame completo da configuração atual do problema, mas apenas a sugestão de algumas hipóteses através da discussão de algumas interpretações recentes: o esbôço de um itinerário cujo traçado definitivo depende de todo um trabalho ainda em curso. Sem esta advertência preliminar, as páginas seguintes poderiam surpreender o leitor: elas devem ser tomadas apenas pelo que são, provisório e simples plano de trabalho. NOTA DA REDAÇĀO - É com o maior prazer que Discurso inclue nêste número um trabalho do Prof. Bento Prado Jr., um dos professores mais brilhantes do Departamento de Filosofia, aposentado em 1969. 
et une politique pourrions-nous dire, ainsi qu'une sociologie de la lecture. Ce texte pourrait servir d'occasion à la formulation de toute une série de questions liminaires: comment Rousseau veut-il être lu, par nous qui appartenons à un autre Siècle, quel est le profil de ce lecteur tardif qu'il attend du fond d'une patience infinie? Comment nous situer, nous mêmes, par rapport à son oeuvre et dans quel lieu la situer pour mieux la lire? Et comme il s'agit d'écrire sur lui — entreprise qui pourrait bien lui paraître dépourvue de sérieux et de fondement - quoi ajouter, dans ce discours second et secondaire, à ce qui est dit par les textes eux-mêmes? Faut-il le situer - nous pensons ici à ceux qui aujourd'hui, répondant à son appel, essayent de réactiver sa pensée ou de la prolonger dans un autre élément - dans la pré-histoire des sciences de l'homme immergé dans un horizon culturel qui ne libérait pas encore leur possibilité ou, au contraire, dans le moment exact où elles s'instituent par l'oeuvre même de son discours, ou encore dans le crépuscule de la métaphysique, qu'il habite encore, mais qu'il aide, sans le savoir, à conduire à son effacement? Marque-t-il le fin d'une époque ou le commencement d'une autre? Voilà bien des questions qu'il ne serait pas étrange de rencontrer à l'introduction d'une étude sur Rousseau: mais des questions qu'il faut maintenant différer, pour pouvoir, plus tard peut-être, bien les poser.

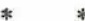

Gardons, pour le moment, seulement cette référence à un lecteur futur, l'idée que l'oeuvre, comme le morceau de sucre de Bergson, doit traverser un certain temps, doit attendre une nouvelle génération pour devenir lisible. M. Gueroult dit, dans l'avantpropos de son Descartes selon l'ordre des raisons, que les différentes générations éclairent, certes, chacune à sa manière, les oeuvres classiques, mais pour ajouter ensuite que "ces jeux d'éclairage laissent le monument intact" (1). Chaque jeu d'éclairage peut mettre en valeur tel ou tel profil aux dépens des autres, mais il ne saurait jamais changer le granulé de la pierre ni l'articulation de ses volumes.

C'est pourtant cette indifférence du monument aux éclairages multiples dont il est susceptible qui semble être mise en cause par le texte de Rousseau; les jeux d'éclairage ne laissent pas le monument intact: ou bien ils le montrent dans toute sa vérité, ou bien ils le cachent - lumière devenue aveuglante - dans sa totalité: et pierre et forme. Le jeu historique des éclairages et des générations semble nous plonger dans uns historicité radicale qui rend problématique l'idée d'une lecture objective telle que la prop̀ose $\mathrm{M}$.

(1) M. Guéroult, Descartes selon l'ordre des raisons, Aubier, 1953, t. I, p. 9. 
Gueroult. Faisant appel, du sein de son siècle, à un lecteur futur et à ses préjugés, à son optique particulière, Rousseau semble anticiper, de son côté même, le dessin d'un cercle herméneutique. Cette historicité ne signifie pas nécessairement relativisme, mais elle fait de l'appartenance du lecteur et du texte à leurs horizons historiques la condition de la compréhension. C'est plutôt la différence entre explication et interprétation qui semble être exclue par cette historicité: la différence qui sépare le commentaire qui redouble le texte de la critique qui le transgresse vers quelque chose qui le dépasse et qui définit, selon J. Derrida, la grille de la "lecture classique". Et si c'est bien à l'intérieur de l'oeuvre de Rousseau que commence à se dessiner la courbe du cercle herméneutique, l'histoire récente de sa lecture semble la fermer et confirmer, ainsi, l'inséparabilité entre explication et interprétation. N'est-il pas, en effet, vrai que nous assistons, dans les dernières décennies à une véritable renaissance de Rousseau, et cette renaissance n'est-elle pas liée aux dernières aventures de la pensée philosophique, l'existentialisme et le structuralisme? Dans cette communication entre le passé et le présent, la notion d'illusion rétrospective est relativisée et perd son sens péjoratif: c'est ce que nous voyons, par exemple, dans un texte où $J$. Derrida parle de l'interprétation de Rousseau et du XVIIIe siècle proposée par Lévi-Strauss:

"Si même on avait le droit de parler d'illusion rétrospective, celle-ci ne serait pas un accident ou un déchet théorique: on devrait rendre compte de sa nécessité et des ses effets positifs. Et cette représentation généalogique de soi est déjá elle-même représentation d'une représentation de soi: ce que le "dix-huitième siècle français", par exemple et si quelque chose de tel existe, construisait déjà comme sa propre provenance et sa présence" (2).

Un de nos buts est de montrer que la distinction entre explication et interprétation est toujours valable et que le lecteur futur auquel Rousseau fait signe n'est pas un élément d'un cercle herméneutique, mais le témoin de la structure même de son discurs, seule preuve de son innocence, et seule chance de salut de ses écrits. Mais il est indéniable que l'inspiration philosophique contemporaine, dans ses formes les plus différentes, a permis une redécouverte de Rousseau et il faut donc commencer par vérifier le poids des "effets positifs" de cette illusion rétrospective qui n'est illusoire qu'apparemment.

Rousseau a bénéficié, en effet, des dernières métamorphoses de la philosophie française et à chaque mutation - existentialisme, structuralisme - on assiste à une sorte de métamorphose de l'oeuvre

(2) J. Derrida, in Cahiers pour l'analyse, $\mathrm{n}^{\circ} 4$, pp. III-IV. 
elle-même. Il suffit d'ouvrir, par exemple, um livre comme la Transformation de la Philosophie Française (3) - où pourtant il n'est jamais question de l'interprétation de Rousseau et où il n'est cité qu'une fois et de manière extrinsèque - pour voir à quel point était fatal une réactivation de sa pensée et comment, dans le choix des thèmes de la nouvelle philosophie, le schèma d'une nouvelle lecture de Rousseau était inscrit comme en filigrane. Bréhier lie, en effet, le style de la nouvelle philosophie à une manière de comprendre et d'éclairer la tradition philosophique; regardons de près un paragraphe significatif:

"Il y a quelques années, un psychanalyste connu vint me voir et me demander ce que je pourrais lui dire sur les rapports de la cure psychanalytique avec les méthodes par lesquelles Plotin, dépassant la conscience immédiate et personnelle, arrivait par approfondissement intérieur à monter au degré où la personne prend racine. Vers les mêmes années et en rapport avec les mêmes questions, des hommes qui n'étaient pas des théologiens lisaient avec ardeur les Pères de l'Eglise et spécialement saint Augustin pour y chercher le secret de cette subjectivité qui, à force de s'approfondir, trouve au plus profond d'elle-même Dieu qui en est le principe. Enfin, je vis l'attention des meilleurs esprits parmi les jeunes se porter sur un philosophe que j'avais autrefois beaucoup étudié, sur Hegel: on trouvait surtout en lui un principe de vie spirituelle, un mouvement dont les faits directement constatés n'étaient que des moments" (4).

L'historien qui, d'habitude, se penche vers une pensée passée se voit ici surpris et pris par le mouvement de la pensée présente; dans son discours se mêlent l'expérience immédiate du temps vécu et l'effort d'objectivation et de réflexion. Ce que ce texte donne à penser c'est justement le lien secret qui noue cette pensée émergeante à un nouveau découpage de la tradition: en se choisissant de nouveaux prob'èmes la philosophie se choisit aussi ses illustres prédécesseurs. Une loi générale de toute lecture du passé? En toute cas, Bréhier montre comment ce nouveau style philosophique découpe à sa manière la continuité de la tradition, dessine sa propre généalogie et constitue sa "bela scuola" - ce qui ne va pas sans introduire un minimum d'inquiétude dans le domaine aparemment calme de l'Histoire de la Philosophie. Ce paragraphe décrit le mouvement par lequel des recherches et des cheminements différents, des entreprises qui se cherchent leur voie dans la solitude et dans l'ignorance réciproque, finissent par se répondre, dans des lieux dif-

(3) E. Bréhier, Transformation de la Philosophie Française, Flammarion, 1950.

(4) Ibid., p. 79. 
férents de la culture et de la science et par produire, sans le savoir et comme à leur sillage, un même collège des Sages qui se réunit au-delà de la dispersion temporelle. Hegel, ou un certain Hegel (5), les Pères de l'Eglise, les gnostiques, sortent de l'oubli, entrent en concile et deviennent des points de repère. Toute une tradition "noire" ou "négative", dualiste ou pluraliste - en tout cas très lointaine du monisme de la Raison et de la positivité de la science - se reconstitue et donne des repères à la pensée, ainsi que le sol sur lequel elle peut s'appuyer pour reprendre son vol. On devine que tout n'est pas rassurant, pour Bréhier, dans le récit qu'il fait de cette métamorphose et on pourrait peut-être parler de quelque chose comme une surprise et une inquiétation devant la transformation dont il se fait le témoin, sinon l'historien: il lui arrive de nous la présenter comme un oubli de la vocation essentielle de l'Occident et de la Philosophie.

Mais porquoi devrait ce nouveau jeu d'éclairage illuminer aussi Rousseau à côte de Hegel et de St. Augustin? La "dialectique de la séparation", tel est le titre du dernier chapitre du livre, du chapitre où Bréhier essaie d'unifier, de donner le fil conducteur de tous les thèmes dont il fait l'inventaire au long de son écrit, dans l'impossibilité de les reconstruire systématiquement. En parlant d'une dialectique de la séparation, Bréhier veut dire que la nouvelle philosophie renonce à l'idéal de dépasser les contradictions qui déchirent l'expérience. Si la philosophie avait pu, auparavant, être définie par l'effort de l'unité ou comme volonté d'unification, on la voit maintenant défine comme l'exploration des écarts infranchissables, des béances du Savoir et de ses limites. Cette idée de la philosophie, qui naît pourtant aussi d'une lecture de Hegel, pourrait être exprimée par l'affirmation que les blessures de l'esprit ne guérissent jamais sans laisser de cicatrices. Une dialectique de la séparation est moins une dialectique qui s'accomplit dans le dépassement des contradictions, qu'une dialectique qui insiste sur leur caractère irréparable, qui ne tourne pas les yeux vers le futur et vers une réconciliation possible, mais vers le passé et vers une unité à jamais perdue. Nous reconnaissons bien aujurd'hui, dans cette thématique de la séparation et de la fracture, une thématique rousseauiste par excellence. On a pu voir dans l'expérience du divorce entre l'être et le pariâtre le ressort de toute réflexion chez Rousseau et la matrice qui se reproduit dans tous les moments de sa pensée. A chaque étape de son oeuvre on peut reconnaître, en effet, le travail d'une dialectique de la séparation et de la rupture: dans la théorie psychologique qu'il propose, dans la genèse des facultés de l'âme à partir de l'unité originaire de la sensation, où chaque progrès est

(5) Par exemple le Hegel de A. Kojève. 
en même temps déchéance; dans sa théorie de la société et de l'Histoire, qui se constitue comme une description de la progresion du mensonge et de la violence à partir de la transparence et de l'unité de la fête et de la société naissante; et enfin dans cette sorte de phénoménologie de l'existence qu'il propose et qui va chercher son secret dans le mouvement qui l'arrache à l'unité de l'Ordre et de la Nature, qui l'oppose à elle-même et qui inscrit dans son intimité une fêlure ineffaçable. Il n'y a pas de doute que le thème de la dialectique de la séparation était fait pour susciter une relecture globale de Rousseau.

Encore il faudrait ajouter que cette dialectique de la séparation ne joue pas essentiellement au niveau de la connaissance et qu'il ne s'agit pas d'une dialectique du Savoir. C'est justement à la limite du savoir, là où il ne peut plus jouer que commence cette dialectique: avec la découverte de la subjectivité ou, plus précisément, de l'intersubjectivité. "Un principe de vie spirituelle", dit Bréhier, en définissant la valeur qu'on commence à reconnaître à Hegel, et non un principe de vie intellectuelle. Il ne s'agit donc pas d'une dialectique qui met en mouvement des termes comme sujet et objet ou concept et expérience: au contraire, le "sujet" y est pris dans et relativisé par une trame de relations dont les autres termes sont autrui et Dieu: le rapport au monde - plus vague, mais antérieur au rapport à l'objet - est lui-même précédé et déterminé par la matrice originaire de l'intersubjectivité. Priorité, donc, du vécu par rapport au connu et privilège philosophique de la dialectique de la conscience. Là aussi nous reconnaissons une affinité essentielle avec la pensée de Rousseau dans la définition du domaine spécifique de l'historicité humaine comme glissement au dehors de la physis et comme instauration d'un domaine autonome qui fait de l'homme une sorte d'effet des systèmes auxquels il appartient. Rousseau se veut, bien sur, plutôt observateur que moraliste:

"Je suis le Botaniste qui décrit la plante. C'est au médicin qu'il appartient d'en régler l'usage" (6).

Mais le style de cette "botanique" de l'homme est défini dans son opposition à la botanique tout court: l'homme, au contraire des autres êtres naturels, est produit par le système des relations qui l'articule aux autres. La connaissance de la nature et la connaissance de l'homme obéissent à des raisons différentes, dont chacune parcourt le chemin inverse de l'autre. Dans un cas, l'individu

(6) $O . C \cdot, \mathrm{I}, \mathrm{p} .1120$ (sauf indication contraire, toutes les références à des textes de Rousseau renvoient à l'édition des Oeuvres Complètes, Pléiade, Gallimard,) . 
est transparent et conduit le regard vers la connaissance de l'espèce: dans l'autre, les individus se modèlent dans leurs rapports réciproques, séparés de leurs corps et de leur espèce:

"La connaissance de la nature des plantes peut bien, par exemple, être aidée par celle du terrain qui les produit, des sucs qui les nourrissent, et de teurs vertus spécifiques, mais jamais on n'en connoitra bien la méchanique et les ressorts si l'on ne les examine en elles même, si l'on n'en considère toute la structure intérieurs, les fibres, les valvules, les trachées, l'écorce, la moële, les feuilles, les fleurs, les fruits, les racines, et en un mot, toutes les parties qui entrent dans leur composition. Dans les recherches morales, au contraire, je commencerois par examiner le peu que nous connoissons de l'esprit humain pris en lui-même et considéré comme individu, j'en tirerois en tâtonnant quelques connaissances obscures et incertaines, mais abandonnant bientôt ce ténébreux labirynthe, je me hâterois d'examiner l'homme par ses relations, et c'est là que je tirerais une foule de vérités lumineuses qui feroient bientôt disparoitre l'incertitude de mes premiers argumens et qui recevroient encore du jour par comparaison" (7).

C'est donc bien au niveau de l'intersubjectivité - ce domaine ouvert par le regard et par "l'opinion" - que l'humanité se constitue et c'est là que son destin est décidé, comme chute au dehors de l'Ordre ou comme espoir de réconciliation: c'est la transparence ou l'opacité de ce réseau qui permet ou empêche l'homme de trouver sa place dans la Nature.

Bréhier insiste aussi, finalement, sur la redéfinition des rapports entre le discours philosophique et la littérature comme un des traits dominants de la nouvelle philosophie. Il y a un effacement de la frontière qui sépare la philosophie de la littérature: elles ne sont plus des régions différentes, distribuées en extériorité, mais des espaces imbriqués internement. Il ne s'agit simplement du fait que, plus qu'auparavant, un même nom propre signe un Traité et un roman ou une pièce de théâtre: c'est le statut même de ces deux langages qui est redéfini et redistribué. Comme le dirait plus tard Merleau-Ponty, la littérature n'est pas simplement une forme régionale de langage, elle est sollicitée par l'Être lui-même (8) et son destin est lié à celui de la vérité. Cette nouvelle complicité entre philosophie et littérature était aussi faite pour jeter une nouvelle lumière sur l"oeuvre de Rousseau. Ce nouveau jeu d'éclairage permet en effet de grouper d'une nouvelle manière les écrits mêmes de Rousseau et de

(7) O. C., II, pp. 1244-5.

(8) Le visible et l'invisible, Gallimard, p. 305. 
compter La Nouvelle Helö̈se, par exemple, parmi les textes "philosophiques": une interprétation nouvelle est rendue possible dans la mesure ou c'est la réalité même de l'oeuvre qui semble avoir bougé.

Enfin, si nous avons évoqué sommairement quelques-uns des traits par lesquels Bréhier dessine le profil de la nouvelle philosophie - telle qu'elle se présentait à la fin des années 40 - c'était pour indiquer autant de signes d'une nouvelle manière de lire Rousseau. De même que Hegel ou St. Augustin, Rousseau commençait à apparaitre comme un de ces penseurs classiques, dans lesquels la pensée contemporaine aime se reconnaître. La lecture de Rousseau devenait solidaire des aventures de la pensée vivante.

\section{II}

\section{L'Existence}

Quand on examine "q'état actuel" de la question J.J. Rousseau, on s'apperçoit, en effet, que l'image traditionelle que nous avions n'est pas sortie intacte de cette métarmorphose. Nous ne nous trouvons par seulemente devant un autre Rousseau - il n'est plus le rhapsode du sentiment, le littérateur brillant qui cache dans la splendeur de son style les contradictions d'une pensée sans profondeur véritable - mais devant un Rousseau - il est maintenant le penseur un peu prophétique qui avance les grands thèmes de la modernité: existence et discours, sociabilité et langage, nature et culture - qui occupe un lieu stratégique dans la pensée du XVIIIe siècle et, même dans l'Histoire de l'Occident (9). On le lit moins avec la curiosité historiographique qui veut reconstruire l'Autre pour le connaître dans son altérité et dans son éloignement qu'avec l'inquiétation de celui qui s'interroge à propos de soi-même à travers la lecture d'un Autre. L'oeuvre n'est plus jugée par un regard qui la transcende et la domine en la survolant: on l'interroge du lieu même d'où elle parle, car il semble qu'elle parle d'ici même; si le nom de Rousseau indique un tournant stratégique de l'Histoire de l'Occident, c'est que ce tournant n'est pas encore clos, qu'il définit un "aujour-d'hui" qui n'est pas encore passé et qui sert toujours d'horizon.

Il est certain que l'oeuvre de Rousseau n'a pas attendu ensevelie dans les limbes de l'histoire les dernières décennies pour être finalement lue et comprise. Faudrait-il rappeler les noms de

(9) J. Cusinier, "L'état actuel de la Question: J.-J. Rousseau", in Informations Littéraires, 1964. 
Kant, de Hegel et de Engels? Dans tous ces cas, il est certain que l'importance philosophique de Rousseau est reconnue et le sens de son oeuvre fixé théoriquement. Aussi, il ne faudrait pas oublier deux siècles d'érudition et d'effort interprétatif, d'historiographie philosophique et littéraire. Mais on ne peut pas non plus s'empêcher de penser à une sorte de refoulement auquel l'oeuvre aurait été soumise pendant longtemps: refoulement ébauché dans sa "mise au ban" par ses contemporains - il nous semble hors de question de mettre en doute aujourd-hui la réalité du "complot" mais perpétué dans les générations suivantes. Que l'oeuvre soit refoulée dans le domaine de la folie ou de la "littérature", il est toujours vrai qu'une certaine idéologie exclut comme dans le cas des idées de totémisme et de hystérie dont parle Lévi-Strauss (10) - une alternative de la pensée dans le monde extérieur du nonsens. L'acharnement polémique contre Rousseau et sa longue vie semble en être la preuve; c'est, il nous semble, ce que suggère Maurice Blanchot dans un essai très intéressant sur lequel il nous faudra revenir plus tard:

"Je ne sais - dit M. Blanchot - si, durant sa vie, Rousseau fut persécuté comme il crut. Mais, puisqu'il n'a manifestement pas cessé de l'être après sa mort, s'attirant les passions hostiles et, jusqu'à ces dernières années, la haine, la fureur déformatrice et l'injure d'hommes aparemment raisonnables, il faut bien penser qu'il y eut du vrai dans cette conjuration d'hostilité dont il se sentit inexplicablement la victime. Les ennemis de Rousseau le sont avec un excès qui justifie Rousseau. Maurras, en le jugeant, s'abandonne à la même impure altération qu'il lui reproche. Quant à ceux qui ne lui veulent que du bien et se sentent d'emblée ses compagnons, nous voyons, par l'exemple de Jean Guéhenno, combien leur est malaisé de lui rendre justice. On dirait qu'il y a en lui quelque chose de mystérieusement faussé qui rend furieux ceux qui ne l'aiment pas et gênés ceux qui ne veulent pas lui faire tort, sans qu'ils puissent parvenir à être sûrs de ce défaut et précisément parce qu'ils ne peuvent en être sûrs" (11).

Si la haine, d'une côté, et le malaise de l'autre ont une même origine, cette origine ne peut être qu'un refoulement, qui précède l'alternative entre le oui et le non, la polémique et l'apologie. Car le refoulement peut se faire, en effet, sous les dehors de l'apologie et de la reconnaissance. Dans les interprétations de Kant (reprises et prolongées par Cassirer) et de Engels, par exemple, Rousseau n'est sauvé qu'au prix d'être assimilé par des systèmes qui

(10) Cf. la première page de Le Totémisme Aujourd'hui, Presses Universitaires de France, 1965.

(11) M. Blanchot, Le Livre à Venir, Gallimard, p. 53. 
lui sont étrangers: on pourrait dire que la lecture kantienne et dialectique de Rousseau transporte le texte dans un domaine où il perd sa spécificité et où il est essentiellement déformé (12). D'un côté, il est celui qui a découvert le monde de la liberté, dont le concept sera fourni par la Critique: le vérité de sa pensée est en un certain sens extérieure à son oeuvre, elle ne peut éclater que dans cette réflexion seconde qui lui trouve un lieu dans le système de la Critique de la Raison. De l'autre, théoricien de l'Histoire, qui découvre la loi ternaire qui conduit, par la médiation de l'inégalité et de la violence, de l'innocence originale et de l'égalité inconsc.ente à l'égalité réfléchie et à la Cité légale, Rousseau devient le précurseur non seulement de Hegel et de la Dialectique, mais aussi de la théorie de la Révolution: le matérialisme dialectique sera l'exposition claire et scientifique de ce qui n'était anticipé que confusément et abstraitement dans l'oeuvre de Rousseau. En montrant comment chacune de ces "synthèses" ferme le sens de l'oeuvre sur une seule de ses lignes de force, Starobinski montre comment elles font disparaître l'étrangeté et l'originalité (13); elle n'est plus ce discours solitaire et fermé sur sa différence, mais un moment essentiel aufgehoben, conservé comme dépassé, en tout cas passé, du devenir de la Raison ou de la construction de la Science. Ce n'est pas seulement 'a tension interne de l'oeuvre qui disparaît, ce aut aut qui fait de l'Emile et du Contrat Social des alternatives différentes dans une même pensée, mais le refus farouche, essentiel chez Rousseau, d'être assimilé par la Philosophie ou le Savoir.

Il a fallu donc un nouveau regard pour rendre à Rousseau son lieu insolite dans l'Histoire de la Philosophie et reconnaitre le caractère philosophique d'un discours qui se tient paradoxalement en dehors de la philosophie. Une nouvelle idée du Savoir, plus souple, et une nouvelle conception des rapports entre philosophie et littérature étaient nécessaires pour que l'oeuvre de Rousseau pût apparaître dans toute sa diversité. C'est l'idée d'existence qui a d'abord permis une nouvelle lecture, dans la mesure où elle a permis de reconsidérer la géographie de l'oeuvre. La tradition, en effet, séparait les textes théoriques des textes littéraires - l'histoire de la philosophie se contennait dans l'Emile, dans les Discours et dans le Contrat Social et laissait le Nouvelle Heloüse à l'historien de la littérature: elle séparait, aussi, les livres "sérieux" des livres "délirants" - quel usage, en effet, faire d'un livre comme les Dialogues? Comment, aussi, des livres comme les Confessions, dans la contingence de leur matière, pourraient intéresser

(12) J. Starobinski, Jean-Jacques Rousseau, La Transparence et l'Obstacle, Gallimard, 1971, pp. 36-49.

Ibid. 
l'historien de la Philosophie? A travers l'idée d'existence on commençait à voir - ce qu'aujourd'hui est établi "qu'il est nécessaire de les entendre (ces écrits autobiographiques) pour entendre dans leur vérité les écrits théoriques, les romans, les pièces de dix années durant lesquelles Rousseau appartient à la République des Lettres”. (14).

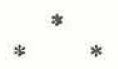

Déjà dans le petit Tableau de la Philosophie Française, Jean Wahl indiquait les chemins d'une lecture alternative de Rousseau Il le présente certes comme le précurseur de Kant; Rousseau, insistant sur la spontanéité du jugement, sur l'activité de la connaissance impliqués dans la "position d'existence" du mot "est", dépasse l'empirisme dans la direction de l'analyse de l'entendement dans la Critique de la Raison Pure, de même qu'il devance la Critique de la Raison Pratique par l'affirmation de l'autonomie et de la liberté comme fondement de la moralité. Mais à côté de l'image du Rousseau qui anticipe la Critique, Jean Wahl commence à ébaucher une autre image:

"Bien plus, aucun philosophe n'est plus près du pur sentiment de l'existence que Rousseau. Il nous décrit ces moments où nous sentons avec plaisir notre existence sans perdre la peine de penser. Variété de la philosophie française! Elle s'était fondée sur la pensée de Descartes: la voici qui se fonde sur un état comme étranger à la pensée. Je pense donc je suis, disait Descartes. Mais dans ces états que nous décrit Rousseau, je suis parce que je pense à peine, on pourrait dire parce que je ne pense pas" (15).

Le "sentiment" dont on parle ici n'est pas le fait d'une subjectivité enfermée dans l'illusion, comme l'âme cartésienne enfermée dans la passion, mais la manifestation d'une expérience qui a une portée ontologique et ouvre l'âme à la vérité: et c'est pourquoi on peut parler d'une sorte de "mystique existentielle". On y reconnaît le lecteur de Malebranche et la description du "sentiment de l'existence" doit être comprise en continuité avec la re-interprétation malebranchienne du cogito. Déjà chez Malebranche, la conscience de soi n'est plus une connaissance claire et distincte, mais une connaissance obscure et confuse, un sentiment. Mais chez Ma'ebranche ce sentiment offre seulement la possibilité de faire le partage entre l'esprit et le corps: la fonctoin du cogito est de nous libérer des illusions qui enferment l'insensé dans son aveuglement

(14) C. Salomon-Bayet, Jean-Jacques Rousseau ou l'unité impossible, Seghers, p. 16 .

(15) Jean Wahl, Tableau de la Philosophie Française, Gallimard, 1962, p. 66. 
à l'esprit. Mais la connaissance ne commence qu'au delà du sentiment, dans la vision en Dieu; dans la formule de M. Gueroult, "Grâce au cogito, nous sommes parvennus à sortir du troupeau des insensés. Grâce à la révélation de son impuissance, nous avons évité d'entrer dans la cabale des superbes" (16). S'il y a quelque chose comme une mystique existentielle chez Rousseau, c'est, au contraire, parce que ce sentiment ouvre déjà la subjectivité vers ce qui la dépasse; il est en lui même une sorte de "vision en Dieu".

C'est bien sur cette idée qu'est centrée l'interprétation que Pierre Burgelin propose dans La Philosophie de l'Existence de JeanJacques Rousseau. Dans l'expression sentiment de l'existence c'est le deuxième terme qui est privilégié: elle ne désigne pas la manière par laquelle l'existence où la réalité se réf'échit à l'intérieur de la subjectivité, comme pourrait supposer une lecture "psychologiste"; c'est bien l'existence elle-même et, avec elle, l'être, qui est dévoilé par et dans ce sentiment. Vision en Dieu? En tout cas, "seul mode d'adhésion à l'être" (17). On peut parler d'une expérience directe de l'être - et non seulement d'un "choc" avec la réalité ou d'une expérience brute - car ce sentiment qui se donne dans le mode du bonheur le saisit comme valeur: dans le sentiment de l'existence la nature se montre comme perfectio ou comme ordo.

Nous assistons donc à un renversement total de la perspective cartésiènne: il ne s'agit plus, dans la découverte de l'intériorité de la conscience de soi, d'une première vérité d'une longue chaîne de raisons. Cette vérité est "la plus concrète et la plus riche" (18): elle peut commencer l'itinéraire de la philosophie, mais seulement à condition que cet itinéraire y vienne aussi finir. Car le philosophe ne l'abandonne jamais à vrai dire: cet étrange cogito est un point de départ, mais il est aussi le sol permanent et le telos de la pensée. Décision absurde du point de vue cartésien, où la vérité du cogito a comme seule fonction de fonder dans l'absolu la connaissance scientifique de la nature. Mais du point de vue de Rousseau, c'est le projet cartésien qui est fou:

"Nous ne connaissons pas une substance dans l'univers, nous ne sommes pas même surs d'en voir la surface et nous voulons l'abîme de la nature. Laissons un si puerile travail à ces enfants qu'on appelle des philosophes. Aprés avoir parcouru la cercle étroit de leur vain savoir il faut finir par où Descartes avoit commencé. Je pense, donc j'existe. Voilà tout ce que nous savons" (19).

(16) M. Guéroult, Malebranche, Aubier, 1955, t. I. p. 35.

(17) P. Burgelin, La philosophie de l'existence de Jean-Jacques Rousseau, Presses Universitaires de France, p. 125.

(18) Ibid.

(19) O. C., IV, p. 1099. 
Mais il faut surtout remarquer que cette expérience privilégiée présente, pour ainsi dire, deux visages et ouvre deux chemins différents. D'un côté, nous l'avons vu, le sentiment de l'existence est la découverte de l'ordre de la nature; de l'autre, il faut voir qu'il est aussi la découverte d'une subjectivité personnelle. $\mathrm{Si}$ ce sentiment ouvre sur l'universel, il ouvre aussi sur une subjectivité singulière: c'est Jean-Jacques qui se rejoint à travers ce cogito . $\mathrm{La}$ découverte de soi n'est pas dissociable d'une vie particulière et des barrières contre lesquelles elle se heurte:

"... la formule ("Je pense, donc j'existe") ne surgit nullement de quelque doute méthodique, mais du désarroi de l'homme en face de la complexité, du déchirement et du malheur de la vie" (20).

C'est ainsi que, dans l'interprétation de P. Burgelin, le sentiment de l'existence nous fournit non seulement le thème central de la réflexion de Rousseau, mais aussi, étroitement liés, les deux pôles entre lesquels elle se meut. L'ordre, d'un côté, et l'existence de l'autre, voilà les deux termes essentiels de la réflexion: plus encore, voilà les deux limites qui définissent le champ de sa réflexion. Les problèmes que Rousseau se choisit, théoriques ou pratiques, renvoient toujours à l'abîme que sépare un terme de l'autre. Le problème philosophique essentiel devient celui de définir les liens qui peuvent conduire d'un terme à l'autre: soit sur la forme de la genèse du mal et de la séparation, soit sur la forme du chemin à suivre pour revenir à l'ordre, pour reconduire l'existence à son lieu dans l'espace de l'ordre:

"Le problème de Rousseau, son problème philosophique au moins, est dans le rapport de c:s deux thèmes qui sont constantement mêlés dans son oeuvre: comment unifier l'ordre et l'existence? L'une me remet à ma modeste place dans le tout sur lequel Dieu règne, dans la cité que régit la loi; l'autre me met au centre. L'une oriente vers une philosophie de la raison, l'autre vers une exploitation du sentiment. Les concilier est le but: je suis fait pour être heureux dans un monde en ordre" (21).

Le sentiment de l'existence ne résout, en effet, ce prob'ème philosophique - il le supprime autant qu'il dure. Dans l'univers que le sentiment instaure ces deux pôles - l'universel et le singulier sont étroitement liés: c'est dans l'expérience directe de soi-même que la conscience singulière se donne l'idée de l'ordre universel et de Dieu qui en est le principe:

(20) P. Burgelin, op. cit., p. 125.

(21) Ibid., p. 572 . 
"De quoi jouit-on dans une pareille situation? De rien d'extérieur à soi, de rien sinon de soi-même et de sa propre existence, tant que cet état dure on se suffit à soi-même comme Dieu" (22).

Il peut sembler paradoxal que cette restriction de la conscience au rapport à soi et cette exclusion de tout ce qui est extérieur puisse avoir un rapport quelconque avec l'universel. Mais c'est le propre de cette extase que de confondre le moi fini avec Dieu et avec le monde. On pourrait dire que le narcissisme de la conscience de soi est le lieu de la manifestation de l'ordre de la nature. Comment en effet ne pas appliquer au Rousseau des Reveries la description du Narcisse faite par G. Bachelard:

"Mais Narcisse à la fontaine n'est pas seulement livré à la contemplation de soi-même. Sa propre image est le centre d'un monde. Avec Narcisse, pour Narcisse, c'est toute la forêt qui se mire, tout le ciel qui vient prendre conscience de sa grandiose image" (23).

Mais si cette découverte individuelle et solitaire de l'ordre ne résout pas le problème théorique ou philosophique, il donne au moins le critère qui pourra le résoudre. Comme le cogito de Descartes - qui n'était pas simplement une vérité mais aussi le critère de toute vérité - la découverte solitaire de l'ordre peut servir de guide à la réflexion. Elle peut, en effet, guider la recherche de l'homme naturel et garantir contre le danger de projeter sur lui les traits de l'homme; en m'excluant du monde de la médiation et de la méchanceté elle me permet de reconstituer l'humanité qui précède la médiation et le mal. On peut bien ainsi lier l'expérience personnelle, dans sa singularité, et le travail théorique dans sa prétention d'universalité: si Rousseau n'était pas l'homme de la nature, s'il n'était pas capable de cette véritable conversion à l'ordre - et telle est, selon lui, sa différence, ce qui fait de lui un autre par rapport à tous les autres - il ne lui serait pas possible de reconstruire conceptuellement et l'image de la première humanité et la génèse du mal qui commande toute l'histoire de l'humanité. Le regard purifié par l'expérience de l'ordre est capable de reconnaître la face originelle et originaire de Glaucus "que le temps la mer et les orages avaient tellemant défigurée, qu'elle ressembloit moins à un Dieu qu'à une Bête féroce..." (24).

Cette sorte d'intuition des valeurs qui, dépassant les obstacles qui enferment l'humanité déchue, rend possible la connaissance du principe et de l'origine, peut aussi guider la tâche pratique et mon-

(22) O. C. I. p. 1047.

(23) G. Bachelard, L'Eau et les Rêves, J. Corti, 1942, p. 36.

(24) O. C., III, p. 122. 
trer le chemin du salut. Parce que son existence personne'le est celle d'un autre et parce qu'elle rend possible la réminiscence du monde idéal et la reconnaissance des valeurs et la présence de Dieu dans la nature, elle rend possible aussi la sagesse pratique: celle qui indique, au sein de la situation présente et particulière, les voies alternatives du salut. Ici, encore une fois, l'universel et le singulier sont combinés: l'universel est présent dans l'idéal d'ordre à rétablir et le singulier dans la situation historique qu'il faut traverser et vaincre pour la rétab'ir. La Politique, la Pédagogie et la Morale seront autant de stratégies commandées par la sagesse et autant de manières de subordonner l'anarchie de la subjectivité et de l'existence à la calme ordonnance de la nature. Au delà des artifices d'un entendement condamné à la sophistique, cette vision des valeurs rend possible la médiation entre l'universel et le singu'ier, entre l'essence et la "situation", et arme la conscience dans sa tâche pratique et théorique:

"Contre ces démons, la voie du salut reste ouverte: pour reconnaître le vrai Dieu selon la Nature il faut que l'homme retrouve son humanité et por cela restaure sa raison. Non l'art d'argumenter, mais la pure vision des valeurs éternelles et des situations concrètes, la raison illuminée par cette voix, à laquelle la société peut nous assourdir tant elle fait de bruit alentour, mais qui ne se tait jamais, nous rappelle à cet ordre, notre fin, toujours à créer par notre sagesse" (25).

L'inspiration platonicienne et l'inspiration "existentiel'e" sont donc en tension, mais non en contradiction, à l'intérieur de ia pensée de Rousseau: l'articulation qui se donnait comme un fait au nıveau de l'expériende singulière de l'extase se reproduit dans la régulation conceptuelle de la théorie. On a pu jouer sur le double sens du génitif de l'expression "la philosophie de l'existence de J. J. Rousseau": l'existence est l'objet de la réflexion de Rousseau ou la réflexion de Rousseau est l'expression de son existence? La dialectique du singulier et de l'universel déployée par la pensée de Rousseau articule aussi les deux sens du génitif: expression d'une existence, cette pensée ne vise autre chose que la subordination de la subjectivité à la vérité de la nature. Quand Rousseau dit: "... il me faut une philosophie pour moi" il pense ensemble les deux choses. - La philosophie de Jean-Jacques est celle qui le soumet à l'ordre en exprimant une existence exemplaire . 
Lire Rousseau est donc lire dans son texte non seulement une théorie, mais l'expression d'un certain rythme existentiel, le destin exceptionnel d'une conscience singu'ière. Tel est aussi le projet de J. Starobinski dans J. J. Rousseau, la Transparence et l'Obstacle. La répétition de quelques thèmes clef donnera la vérité et de ce discours et de cette existence: tels les thèmes de la transparence et de l'obstac'e. Au dessus des concepts construits, dans le "labyrinthe fangeaux" des récits autobiographiques, dans la fiction, on redécouvre la permanence de quelques images qui doivent donner la loi de l'oeuvre, ainsi que l'intelligibilité de la vie. Entre l'analyse thématique et la psychanalyse existentielle, le commentaire de J. Starobinski nous fournit une lecture originale de Rousseau.

Mais que'le est la force herméneutique des images de la transparence et de l'obstacle? Comment les images de visibilité et de voile peuvent nous instruire à propos de la pensée de Rousseau? Tout d'abord, le récit autob ographique des Confessions dans le langage choisi, aiguille le lecteur vers des catégories particulièrement surdéterminées. Ces catégories semblent naître de la remémoration de l'existence et être solidaires d'un effort d'auto-compréhension et d'auto-justification. Ecoutons un texte du commencement des Confessions:

"Nous restâmes encore à Bossey quelques mois. Nous y fûmes comme en nous représente le premier homme encore dans le paradis terrestre, mais ayant cessé d'en jouir: c'était en apparence la même situation, et en effet une toute autre manière d'être. L'attachement, le respect, l'intimité, la confiance ne liaient plus les élèves à leurs guides; nous les regardions plus comme des dieux qui lisaient dans nos coeurs: nous commencions à nous cacher, à nous mutiner, à mentir. Tous les vices de notre âge corrompoient notre innocence et enlaidissoient nos jeux. La campagne même perdit à nous yeux cet attrait de douceur et de simplicité qui va au coeur: elle nous semblait déserte et sombre, elle s'était comme couverte d'un voile qui nous en cachait les beautés. Nous n'allions plus grater légèrement la terre et crier de joye en découvrant le germe du grain que nous avions semé. Nous nous dégoutames de cette vie: on se dégouta de nous; mon oncle nous retira, et nous nous séparames de $\mathbf{M}$. et Mlle. Lambercier ressasiés les uns dis autres, et regrettant peu de nous quitter" (26).

L'épisode du peigne brisé n'est à première vue qu'un événement de l'enfance de Rousseau, mais cet épisode se revêt immédiatement d'un poids symbolique qui le dépasse et qui finit par quali-

O. C., I, pp. $20-1$. 
fier l'existence dans sa totalité. Cet événement marque la fin de la "sérénité de ma vie enfantine" et le commencement de ce destin particulier L'enfance est définie comme l'espace d'un jeu innocent dans une nature transparente, sous le regard bénévole des dieux: l'enfant glisse sur l'apparence, "grate légèrement la terre", qui ne cache aucun fond caché et le bonheur de ce jeu "superficiel" est confirmé par le regard des dieux qui ne creuse aucun secret au delà du visible. C'est cette visibilité ou cette publicité totale des regards qui trouve sa fin et sa négation avec l'épisode du peigne brisé. A ce moment, l'enfant découvre dans le malheur quil y a de l'invisible, car son innocence échappe au regard supposé omniscient des dieux, qui cessent, du même coup, de l'être. L'expérience enfantine est le sol et l'humus de la pensée: le thème assez courant de la différence entre l'être et l'apparence est nourri, chez Rousseau, par la force de cette expérience à jamais vivante. Et le schéma de l'expérience vécue servira de modèle à la réflexion théorique: c'est ce voile qui s'introduit entre les âmes et qui empêche, aussi, l'accès à la nature, qui commence à sembler "déserte et sombre... couverte d'un voie qui nous en cachait les beautés", c'est ce même voile qui sera invoqué, au niveau de la théorie, pour rendre compte du passage de la bonne nature à la méchanceté de la société. J. Starobinski insiste sur l'isomorphisme entre cette dialectique de l'être et de l'apparence, de cette découverte de l'injustice et de la violence, de l'impuissance de la persuasion de la conscience innocente, et la dialectique déployée dans les Discours:

"Il vient d'apprendre que l'intime certitude de l'innocence est impuissante contre les preuves apparentes de la faute; il vient d'apprendre que les consciences sont séparées et qu'il est impossible de communiquer l'évidence le plus immédiate que l'on éprouve en soi-même. Dès lors, le paradis est perdu; car le paradis, c'était la transparence réciproque des consciences, la communication totale et confiante. Le monde lui-même change d'aspect et s'obscurcit. Et les termes dont Rousseau se sert pour décrire les conséquences de l'incident du peigne cassé, ressemblent étrangement aux mots par lesquels le premier Discours dépeint le "cortège des vices" qui fait irruption dès qu'on n'ose plus paraître ce qu'on est"

(27)

Dès que l'innocence est devenue un secret, toute l'existence devient secrète: pour celui qui a été accusé injustement, rien ne reste sinon une ressource: se cacher. Si seules les apparences ont du poids, il faut se créer l'apparence nécessaire, sans se montrer dans l'immédiat. Si le regard du "spectateur" est devenu aveugle

J. Starobinski, op. cit., p. 19. 
aux évidences du coeur innocent, la nature elle-même devient invisible à tout regard et au monde entièrement superficiel et visible du paradis se substitue un univers des profondeurs (où on ne gratte plus la terre, mais où on cherche à ses entrailles) où tout est voilé: se cacher est en même temps cacher la nature, l'ordre et Dieu:

"On ne peut réfléchir sur les hommes, qu'on ne se plaise à se rappeler l'image de la simplicité des premiers temps. C'est un beau rivage, paré des seules mains de la nature, vers lequel on tourne nécessairement les yeux, et dont on se sent éloigner à regret. Quand les hommes innocents et vertueux aimaient à avoir les dieux pour témoins de leurs actions, ils habitaient ensemble sous les mêmes cabanes; mais bientôt, devenus méchants, ils se lassèrent de ces incomodes spectateurs" (28).

Le parallélisme entre les textes est évident: Rousseau utilise, pour décrire sa découverte de l'injustice dans l'enfance, le langage des textes théoriques où il décrit la naissance de l'injustice dans l'Histoire de l'espèce.

La coïncidence des images et du langage ne renvoie pas simplement à un parallélisme entre les manières de décrire le destin personnel et le destin de l'humanité, mais aussi au secret même de l'oeuvre, à ce lieu où s'articulent les deux genres d'écrits. C'est un lieu qui est lui-même extérieur à l'oeuvre et précède le projet d'écrire. La lecture doit dépasser l'oeuvre vers le silence qui la précède et où elle puise sons sens le plus profond. Car l'expérience de la rupture est aussi l'expérience qui explique le projet même d'écrire: pour Rousseau, explique J. Starobinski, écrire devient nécessaire justement avec l'expérience de l'impossibilité de la communication immédiate. S'il est vrai qu'un voile cache les évidences du coeur innocent, il faut fuir, se cacher sous le masque de l'Auteur: l'écriture est le moyen qui, en supprimmant l'immédiat, rend possible un retour futur à l'immédiation. L'oeuvre n'est donc qu'une médiation éphémère entre deux silences, la solitude provisoire de quelqu'un qui, ayant perdu le paradis, aspire toujours à y revenir. Et si finalement Rousseau est condamné à la solitude, s'il est à jamais emprisonné dans son oeuvre, se n'est pas par sa décision, mais par l'"oeuvre" du voile et de l'obstacle que le complot des Messieurs finit par jeter entre lui et l'humanité. C'est le même schéma, il est curieux de le noter, utilisé par Proust pour définir la trajectoire dElstir: 
"Et sans doute, les premiers temps, avait'il pensé, dans la solitude même, avec plaisir que, par le moyen de ses oeuvres, il s'adressait à distance, il donnait uns plus haute idée de lui, à ceux qui l'avaient méconnu ou froissé. Peut-être alors vécut-il seul, non par indifférence, mais par amour des autres, et, comme j'avais renoncé à Gilbcrte pour lui réapparaître un jour sous des couleurs plus aimables, destinait-il son oeuvre à certains, comme un retour vers eux où, sans le revoir lui-même, on l'aimerait, on l'admirerait, on s'entretiendrait de lui; un renoncement n'est pas toujours total dès le début, quand nous le décidons avec notre âme ancienne et avant que par réaction il n'ait agi sur nous, qu'il sagisse du renoncement d'un malade, d'un moine, d'un artiste, d'un héros. Mais si'l avait voulu produire en vue de quelques personnes, en produisant il avait vécu pour lui-même, loin de la société à laquelle il était devenu indifférent; la pratique de la solitude lui en avait donné l'amour, comme il arrive pour toute grande chose que nous avons crainte d'abord, parce que nous la savions incompatible avec de plus petites auxquelles nous tenions et dont elle nous prive moins qu'elle ne nous détache. Avant de la connaitre, toute notre préoccupation est de savoir dans quelle mesure nous pourrons la concilier avec certains plaisirs qui cessent d'en être dès que nous les avons connus" (29).

C'est bien le même itinéraire de Rousseau, tel que Starobinski nous le reconstitue, à partir du projet premier d'écrire pour reconquérir la présence immédiate sous les yeux bénévoles des "dieux", jusqu'à la solitude finale et calme des Rêveries; la solitude provisoire a découvert la valeur absolue de la solitude et devient, envers autrui, absolument indifférente comme si elle était dans une "planète étrangère".

\section{Le Langage}

La vogue plus récente du "structuralisme" n'a pas, non plus, laissé intact notre rapport à Rousseau: elle a suscité une lecture de Rousseau à travers l'axe du langage. Ce n'est plus autour de l'idée d'existence qu'on fait tourner la totalité de l'oeuvre, mais autour de celle des signes et du discours. Dans cette nouvelle "transformation de la philosophie française", on peut reconnaître un changement radical dans le rapport aux classiques: au lieu de He-

(29) M. Proust, A l'ombre des jeunes filles un fleurs, Le Livre de Poche, p. 420. 
gel et de St. Augustin, ce sont les auteurs du XVIIIe siècle qui fournissent le paradigme de la raison philosophique; dans le langage de M. Serres, c'est la fin de l'interprétation romantique (30). Il s'agit, dans ce nouveau idéal de rationalité, aussi d'un nouveau découpage de la tradition. Et dans ce nouvel élément Rousseau ne perd pas la place qu'il avait conquise auparavant. Au contraire, il bénéficie encore, non seulement de la réactivation de la pensée du siècle des Lumières, mais il garde toujours dans cet horizon une place stratégique et essentielle. La différence même qui l'oppose aux "philosophes" est quelque chose d'essentiel au siècle des philosophes. Comme si la vérité des Lumières ne pouvait apparaître que là où elles trouvent la théorie de leurs limites.

Il n'apparaît plus, dans cette nouvelle bibliographie, comme le penseur de la tragédie ou comme le phénoménologue du mit-sein, mais comme le précurseur de toute une nouvelle réflexion sur le langage et sur les rapports entre nature et culture. Une sorte de complicité s'établit entre la pensée contemporaine et la pensée du XVIIIe siècle au point qu'on puisse dire que la première répète la deuxième dans le geste même où elle affirme son originalité:

"Le motif initial de notre propos est la réactivation par l'anthropologie moderne d'un geste caractéristique de la philosophie du XVIIIème siècle: celui par lequel, pour expliquer la genèse et la fonctionnement de la pensée dans l'individu, on se contraint de recourir à une première origine de la culture. Cette attitude ne pouvait surprendre tant que, sereinement adossée aux dogmes de l'Aufklärung

et du positivisme, l'ethnologie identifiait, explicitement ou non, primitivisme et infantilisme. Le sauvage et l'enfant constituant alors, conformément à la tradition empiriste, deux figures parallèles des éléments premiers et simples de la pensée, le "primitif", puer robustus du monde théorique, semblait devoir donner des premicrs commencements une image plus sensible et plus pure. On pourrait au contraire s'étonner de voir cette référence à des formes de culture plus originaires survivre au naufrage de la "mentalité primtive", si C. Lévi-Strauss n'avait lui-même écarté l'ambiguité en indiquant, au-delà ou en face de Condorcet et de l'idéologi乞 des progrès de l'esprit humain (ou inversement d'une exaltation naïve de l' "homme naturel"), une autre pensée de Y'origine: celle de Rousseau"' (31).

Il est toujours donc à la limite de la modernité et son oeuvre aide son accouchement. Mais c'est l'idée même de modernité

(30) M. Serres, Hermès ou de la Communication, Minuit.

(31) J. Mosconi, in Cahiers pour l'Analyse, n ${ }^{9}$, p. 47. 
qui a changé et, avec elle, l'idée de l'âge classique; la modernité n'est plus définie comme le lieu de la découverte de la finitude et de la subjectivité, elle est désormais pensée comme retour à une idée de la raison et du langage qui avait été refoulée par le XIXe siècle. A la psychologie "dramatique" de 1" interprétation romantique", on oppose une psychologie associationiste qui dissout lâme dans le langage: le lieu stratégique ne se situe plus dans le rapport de la conscience avec soi, avec autruit et avec Dieu, mais dans ce lieu où le langage et la société construisent et produisent la conscience dans sa plus profonde intimité. "La langue est une raison qui a ses raisons et que l'homme ne connaît pas", dit LéviStrauss. Le XVIIIe siècle n'est pas aussi pensé comme le moment où l'intellectualisme commence sa crise, où la sensation, l'imagination commencent à recupérer leur vrai sens, mais comme le moment d'une première ébauche de critique de la métaphysique, comprise comme philosophie de la représentation et de la conscience, L'idée d'ordre, libérée de son fondement dans l'intériorité du cogito, anticiperait l'idée de structure, comme le lieu où le sens n'a pas besoin de la conscience pour venir à l'être.

Cette nouvelle bibliographie a certes des points communs, une certaine optique et un certain choix de textes et de thèmes, un certain langage commun, mais elle n'est pas nécessairement convergente. Si Rousseau est toujours pensé comme quelqu'un qui nous conduit aux limites de la métaphysique, c'est l'idée même de métaphysique qui n'est pas univoque: autant de conceptions de la métaphysique, autant de lectures différentes de Rousseau. C'est ainsi, par exemple, que nous trouvons chez LéviStrauss et chez J. Derrida deux lectures rivales de la pensée de Rousseau.

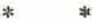

Ce que Lévi-Strauss nous invite à lire chez Rousseau c'est bien une révolution à l'intérieur de l'histoire de la pensée occidentale. Non plus la découverte de la finitude ou de la dialectique de l'intersubjectivité, mais cette révolution qui dénonce, à travers une certaine idée de la raison, la persistence des préjugés de l'enfance ou des illusions de la conscience de soi, et qui annonce la mort de la philosophie, de tout savoir qui se fonde sur l'opposition entre le sensible et l'intelligible. Lévi-Strauss reconnaît, dans les textes où Rousseau fait le procès de la philosophie, la même démarche qui commande la critique ethonologique de la 
métaphysique: dans un cas comme dans l'autre, le projet d'universalité de la philosophie apparaît comme idéologique. Qu'il s'agisse de l'Illustration ou de la Dialectique, ce qui s'affirme dans cette apparente volonté d'universalité c'est plutôt la particularité d'une conscience historique et locale: volonté pervertie de l'homme du monde ou volonté utopique de l'homme de gauche. Dans les deux cas, ce qu'on oppose à la philosophie, c'est une rationalité capable d'illuminer le concret - dans la couture du sensible et de l'intelligible - et qui peut même, dans des conditions "rares et précieuses", vibrer dans la perception et se répercuter dans le corps. Dans les deux cas, la vision d'un paysage sauvage fournit le monogramme de cette raison susceptible d'une usage "esthétique". En effet, comment ne pas rapprocher de certaine textes des Rêveries, où l'extase est la Stimmung de la découverte de l'ordre de la nature, le beau paragraphe des Tristes Trqpiques:

"Que le miracle se produise, comme il arrive parfois: que, de part et d'autre de la secrète fêlure, surgissent côte à côte deux vertes plantes d'espèces différentes, dont chacune a choisi le sol le plus propice; et qu'au moment même se devinent dans la roche deux ammonites aux involutions inégalement compliquées, attestant à leur manière un écart de quelques dizaines de millénaires: soudain l'espace et le temps se confondent: la diversité vivante de l'instant juxtapose et perpétue les âges. La pensée et la sensibilité accèdent à une dimension nouvelle où chaque goutte de sueur, chaque flexion musculaire, chaque halètement deviennent autant de symboles d'une histoire dont son corps reproduit lø mouvement propre en même temps que ma pensée en embrasse la signification. Je me sens baigné par une intelligibilité plus dense, au sein de laquelle les siècles et les lieues se répondent et parlent des langages enfin réconciliés" (32).

On y voit une même expérience de la continuité entre intelligence et sensibilité, une même expérience de la suppression du temps. Mais aussi une même découverte des limites de la Philosophie. Il s'agit moins d'une critique d'une certaine philosophie que d'un refus de toute représentation "philosophique" de l'homme, c'est-àdire, de toute perspective qui le vise dans son identité ou dans son intériorité et qui oublie de le chercher dans le mouvement par lequel il se détache de l'autre que lui. Il fallait que le cogito meure, dissouts par l'analyse d'une psychologie associationiste et par la réflexion sur l'Autre; il fallait qu'il soit décentré de sa pure immanence, pour que l'homme pû apparaître à la lumière du Savoir. L'Archéologie des sciences humaines retrouverait le geste inaugural de ces sciences dans le refus du partage fondamental de

(32) L.-Strauss, Tristes Tropiques, Union Générale d'Editions, p. 43. 
la métaphysique cartésienne; geste d'accueil autant que de refus, qui donne à voir l'appartennance de l'homme à un ordre qui le précède. Par ce geste, "Rousseau ne s'est pas borné à prévoir l'ethnologie: il l'a fondée" (33).

Dans sa lecture de Rousseau, Lévi-Strauss parcourt les divers niveaux où s'opère ce décentrement fondamental: critique psychologique du cogito, critique de l'ethnocentrisme, critique de l'humanisme. Dans tous ces niveaux, c'est le même mouvement qui se reproduit: celui qui conduit le soi de son noyau à sa périphérie. D'abord, en remplaçant le cogito par le sentiment de l'existence: lecteur de Malebranche, nous l'avons déjà vu, mais aussi lecteur de Condillac, Rousseau transforme la conscience de soi dans une expérience et dans une connaissance confuse. Le Vicaire disait:

"J'existe et j'ai des sens par lesquels je suis affecté. Voilà la première vérité qui me frappe, et à laquelle je suis forcé d'acquiescer. Ai-je un sentiment propre de mon existence ou ne la sens-je que par mes sensations? Voilà mon premier doute, qu'il m'est, quand à présent, impossible de résoudre" (34).

Ce texte ouvre deux possiblitée d'interprétation de la critique rousseauiste du cogito cartésien. S'il est vrai que j'ai "un sentiment propre de mon existence", le sentiment est, comme l'indique P. Burgelin, essentiellement différent de la sensation et établit une sorte de communication directe et interne de soi à soi: plus encore, cette intériorité devient le lieu du dévoilement de l'Etre; Rousseau est alors surtout lecteur de Malebranche. Si, au contraire, le sentiment de l'existence suppose toujours la sensation, comme l'indique Lévi-Strauss (thème présent aussi dans la Pensée Sauvage, dans la critique de la théorie sartrienne de la temporalité), il n'est que la preuve de l'intellectualisme et de l'objectivisme de Rousseau: l'illusion de l'intériorité n'est que l'epiphénomène de l'extériorité et Rousseau est surtout lecteur de Condillac. La conscience de soi aurait donc une nature essentiellement centripète: elle ne s'établirait que par la médiation de la sensation. Plus encore, l'identité constituée par le sentiment de l'existence serait toujours en sursis: elle ne dépasserait jamais la flamme de l'instant et ne pourrait assurer la continuité temporelle du moi. L'âme est plus changeante que Protée ou qu'un Caméléon et on peut dire: "Rien n'est si dissemblable à moi que moi-même" (35). Lévi-Strauss insiste sur le caratère "objectiviste" de cette psychologie: il ne s'agit plus

(33) L.-Strauss, "J.-J. Rousseau, fondateur des sciences de l'homme", in Jean-Jacques Rousseau, publié par l'Université Ouvrière et la Faculté de Lettres de l'Université de Genève, Neuchatel, 1962, p. 240.

(34) O. C., IV, pp. 570-1.

(35) O. C., I, 108. 
de signaler en moraliste "l'inconstance de l'humaine nature" et de "peindre le passage", mais en homme de science de donner les raisons objectives de cette inconstance. C'est Rousseau qui le dit: “... j'avois un objet plus neuf et même plus important. C'étoit de chercher les causes de ces variations ..." (36).

Mais si le centre de gravité de la conscience est ainsi déplacé par l'analyse psychologique, ce déplacement est confirmé par la comparaison ethnologique. C'est en ce sens que Lévi-Strauss commente la formule de Rousseau: "Quand on veut étudier les hommes, il faut regarder près de soi; mais pour étudier l'homme, il faut apprendre à porter sa vue au loin". C'est la démarche même de la connaissance ethnologique qui est exprimée dans cette formule qui montre, au delà de l'égocentrisme et de la naïveté, que toute humanité est locale et que l'universalité ne se trouve que dans le système des différences. Egocentrisme et naïveté qui n'attendent pas la rencontre de l'identique pour se montrer au grand jour, et qui se révèlent déjà à l'intérieur d'une même société, dans l'antagonisme des groupes qui la composent. St. Preux définit cette attitude: "Avoir un Carrosse, un Suisse, un maître d'hôtel, c'est être comme tout le monde. Pour être comme tout le monde il faut être comme très peu de gens" (37). Narcissisme stratégique, car il garantit la bonne conscience et l'identification à soi d'une humanité particulière: un regard excentrique pourrait donner l'occasion d'une visite au sous-sol inquiétant de cette conscience et de la découverte du même la sous surface confortable des oppositions.

Cette double réduction se parfait dans une décentration de l'humanité prise globalement, qui la replonge dans une promiscuité avec la vie en général, que la tradition métaphysique avait refoulée. Métaphysique, égocentrisme et humanisme se superposent. Cette solidarité avec la vie apparaît dans l'idée de piété. La piété, souligne Lévi-Strauss, n'est pas seulement la forme de l'identification avec l'humanité en général: par elle, l'homme redécouvre le sous-basement vital de son existence. C'est sur cette faculté primordiale que viendront se dessiner, dans un jeu d'oppositions, les prédicats que la science doit déchiffrer (38). L'homme s'identifie d'abord par la piété avec la totalité du vivant pour se distinguer, ensuite, à l'intérieur de ce champ, du non-humain.

Cette série de réductions qui finit par la découverte d'une logique inscrite dans le sensible, et d'une pensée anonyme qui pré-

(36) O. C., I, p. 408.

(37) O. C., II, p. 252.

(38) Le Totémisme Aujourd'hui, p. 145. 
cède le sujet est liée, chez Rousseau, à une certaine conception du langage, de sa nature et de sa genèse: l'analyse du langage, aussi, nous reconduit à une couche oubliée et primitive - le langage de la métaphore - qui est l'origine du langage de la raison. Si Rousseau a pu détruire la métaphysique du cogito, c'est parce qu'il a su lui poser des problèmes qu'elle ignorait et auxquels elle ne pouvait pas survivre. Des problèmes où "... des préocupations, pour lui si impérieuses, bien qu'elles fussent à première vue étrangères au labeur du philosophe et de l'écravain; je veux dire le linguistique, la musique et la botanique" (39). Rousseau est, pour Lévi-Strauss, le nom propre qui indique, dans la découverte de l'épaisseur du langage, la fin de la métaphysique, de la pensée ct de la conscience.

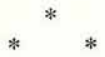

Chez J. Derrida aussi (40) Rousseau - ou ce qu'il appelle l'époque de Rousseau - est pensé sur le fond de l'histoire de la métaphysique: lire Rousseau est, en même temps, penser le problème de la fin de la métaphysique. Mais la continuité entre les deux lectures n'est qu'apparente: le mot métaphysique ne saurait avoir le même sens dans les vocabulaires de Lévi-Strauss et de Derrida. Pour Lévi-Strauss, la métaphysique ne peut avoir que le sens pégatif d'un système de préjugés qui fonctionne comme obstacle épistémologique à l'institution de la connaissance scientifique. Comme la raison galiléenne a dû détruire la métaphysique aristotélicienne du monde perçu pour rendre possible la connaissance scientifique de la nature, la raison structurale doit détruire la métaphysique cartésienne de la conscience pour rendre possible la connaissance scientifique de l'homme. Pour Derrida, au contraire, le destin de la métaphysique - ou de ce qui est en jeu dans et par la métaphysique - ne peut pas être décidé dans l'élément de la scientificité: car l'idée même de science n'est pas indépendante ou extérieure à l'"époque de la métaphysique". C'est chez Heidegger que nous trouvons le modèle de cette conception de la métaphysique: commentant un texte de Hegel (41), Heidegger dit:

"Le premier paragraphe nomme ce qui est en cause dans la philosophie: "Elle considère le présent en sa présence, et (considère)

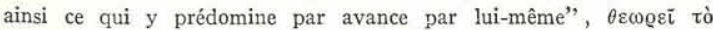

(39) L.-Strauss, "J.-J. Rousseau, fondateur des scinces de l'homme, p. 244.

(40) J. Derrida, De la Grammatologie, Minuit, 1967.

(41) M. Heidegger, "Hegel et son concept dexpérience", in Chemins qui ne mènent nulle part", Gallimard, 1962, tr. Wolfgang Brokemeier. 


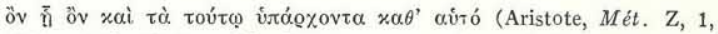
1003 a 21). Cette prédominance concerne le venir à paraître dans l'éclosion. La philosophie considère le présent dans sa présence. Cette considération envisage le présent. Elle le vise de telle sorte qu'elle ne r:garde le présent qu'en tant que tel. La philosophie regarde: le présent quant à son aspect. Dans la vue de ce regard, aucune profondeur abstruse ne prend ses ébats. La $\theta \varepsilon \omega \varrho i ́ \alpha$ est le désenchantement de toute connaissance. Hcgel dit, dans la langue de sa pensée: la philosophie est "la connaissance effective de ce qui est en vérité". Entretemps, ce qui est en vérité, le vraiment étant, s'est mis en évidence comme le réel dont l'effectivité est l'Esprit. Or, l'essence de l'Esprit réside en la Conscience de soi"' (42).

Ce "entretemps" dont parle Heidegger, le inzwischen qui sépare la définition aristotélicienne de la définition hégélienne, définit aussi bien la nature et l'histoire de la métaphysique. Ce texte nous dit, il est vrai, la différence entre Aristote et Hegel: "entretemps" le "vraiment étant" a changé de visage et est devenu la présence à soi de la consc:ence, repraesentatio ou Savoir Absolu; la repraesentatio efface l'eidos comme l'ousia et se met à leur place, même si, comme le dit Heidegger ailleurs, l'eidos peut être pensé comme la condition première de l'avènement du "vraiment étant" comme représentation:

"En revanche, que pour Platon l'étantité de l'étant se détermine comme Eĩoos (ad-spect, "vue"), voilà la condition lointaine, historiale, souveraine dans le retrait d'une secrète médiation, pour que le Monde (Welt) ait pu devenir image (Bild)" (43).

Mais, au delà de la différence indiquée, le texte marque l'identité et la permanence: que le vraiment étant soit ousia ou conscience de soi, il est toujours vrai que le vrai est pensé comme présent; l'essence de la métaphysique, inaltérée d'Aristote à Hegel, réside dans la décision d'identifier le vrai avec le présent en tant que présent, das Anwesende als das Anwesende. Le temps et l'Être sont ainsi pré-déterminés dans cette définition de la "Sache der Philosophie": le temps est réduit à la dimension de présent et l'être est déduit à l'étant qui se donne "devant les yeux".

A cette structure, Derrida ajoute une autre détermination: ce privilège de l'étant et du présent est aussi le privilège de la "parole vivant". la métaphysique n'est pas caractérisée, ainsi, comme limitation à l'étant seulement, mais aussi comme limitation au logos. $\mathrm{Si}$ Heidegger parlait d'un oubli de l'être, Derrida indique quelque

(42) Ibid., p. 109.

(43) Ibid., p. 82. 
chose comme l'oubli de l'écriture: la métaphysique est indissociablement refoulement de la différence entre l'étant et l'être et de l'espacement et de la trace qui, précédant la voix, la rend possible. L'écriture n'est pour la métaphysique que le dehors du langage, l'ombre du logos dans le monde de l'empirique et de l'inessentiel.

Il appartient donc à l'essence de la métaphysique de passer le signe écrit sous silence. Et c'est parce que la métaphysique doit nécessairement oublier l'écriture que la réflexion de Rousseau est le signe d'une limite: son discours, parce qu'il prend l'écriture pour objet, peut être compris comme le lieu où la métaphysique touche sa propre limite. Il ne s'agit pas simplement du fait de prendre l'écriture - l'extériorité et l'au-delà du logos - comme objet du réflexion: en prenant l'écriture comme objet et en la définissant comme supplément de la parole, Rousseau est conduit à brouiller les claires oppositions qui sont au fondement de la métaphysique. Dans la dialectique qu'il institue entre l'originaire et le supplément qui prend sa place, Rousseau affirme la plénitude de la parole et de la présence mais, en même temps, il renverse les termes: la parole peut être le lieu de l'absence et l'écriture peut garantir le retour de la présence. C'est la "logique" même qui structure le discours de la métaphysique qui est en danger dans ce renversement:

\footnotetext{
"Ayant d'une certaine manière, disions-nous, reconnu cette puissance qui, inaugurant la parole, disloque le sujet qu'elle construit, l'empêche d'être présent à ses signes, travaille son langage de toute une écriture, Rousseau est néanmoins plus pressé de la conjurer que d'en assumer la nécessité. C'est pourquoi, tendu vers la reconstitution de la présence, il valorise et disqualifie à la fois l'écriture. À la fois c'est-à-dire dans un mouvement divisé mais cohérent. Il faudra tenter de ne pas en manquer l'étrange unité. Rousseau condamne l'écriture comme destruction de la présence et comme maladie de la parole. Il la réhabilite dans la mesure où elle promet la réappropriation de ce dont la parole s'était laissé déposséder. Mais par quoi, sinon déjà par une écriture plus visible qu'elle et déjà installée dans sa place?" (44).
}

En faisant ainsi passer la présence dans l'absence et l'absence dans la présence, c'est le système de la "conceptualité classique" qui entre en crise: si l'entreprise de Rousseau appartient encore à la stratégie de refoulement de l'écriture et de la différence, de défense de l'identité et du logocentrisme, son itinéraire le conduit dangereusement dans le monde du dehors. Le "rêve" de Rousseau est bien de faire le "supplément" rentrer dans l'originaire et de

(44) J. Derrida, op. cit., p. 204. 
discipliner l'écriture dans l'élément de l'identité, mais il finit par indiquer la maladie secrète qui hante la parole et par suggérer que la pleine présence à soi peut être une illusion de la conscience. Le résultat de la réflexion de Rousseau, qui peut ne pas coïncider avec le but visé et avec l'intention - ici une différence s'inscrit entre la vouloir dire et le dire - est l'idée paradoxale d'un "supplément originaire", signe d'une pensée qui n'etait pas encore représentable dans l'élément de la métaphysique. Rousseau est, pour J. Derrida, penseur de la métaphysique qui, dans l'effort de refouler l'écriture, donne à penser la radicalité et le caratère originaire de l'écriture.

\section{IV}

\section{L'excentricité de l'oeuvre ou l'impossible théorie}

Dans l'introduction de son Système de Leibniz, M. Serres s'en prend au problème du cercle que unit la vérité epistémologique ̀̀ la vérité historique et de la réactivation, par les révolutions de la raison scientifique, des vérités oubliées de l'histoire. La tentation est grande, en effet, de parler d'illusion rétrograde; l'interêt des analyses de Mi. Serres est justement de montrer la positivité de ces récurrences et de ces recouvrements historiques. On pourrait même voir, dans ces commentaires récourrents qui sont faits essentiellement au futur, l'explicitation de quelque chose qui était en germe dans le texte commenté:

"On serait tenté de décrire un intervalle historique où le début leibnizien mime et prévoit la fin contemporaine, en dessinant l'entredeux comme le développement partiel et lacunaire de la construction initiale, et l'approximation lacunaire et partielle de l'architecture terminale. Et à mesure que l'on s'approche de celle-ci, dans la même mesure on développe celle-là. Le système leibnizien est de mieux en mieux centré à mesure que l'on s'approche des mathématiques contemporaines (le commentaire et ses variations le montrent), parce que celles-ci s'instaurent à la manière et dans le style de celui-là, en s'épurant progressivement, en remontant vers leur source formelle et en supprimant les privilèges traditionnellement accordés à telle ou telle région singulière de leur savoir technique" (45).

Ne serait-on pas tenté de dire autant de l'histoire du commentaire de Rousseau? N'est-il pas vrai de Rousseau aussi qu'il jouit

(45) M. Serres, Le système de Leibniz, Presses Universitaires de France. 
d'une situation paradoxale "parmi ses contemporains, par rapport à ses devanciers et au regard de ses successeurs"? Ne pourrait-on dire aussi de Rousseau qu'il "est de son temps et paraît à beaucoup d'égards la plus fine expression des thèses de son époque", mais qu'il "les dépasse tous, et selon l'histoire, il annonce des jours nouveaux"? On doit reconnaître, au moins, que cette situation paradoxale parmi ses contemporains et au regard de l'histoire est un des thèmes de la pensée de Rousseau. Il écrit, certes, à l'intérieur de son siècle et s'adresse à lui; nous voulons ainsi signifier simplement qu'il est pris dans la trame d'une structure historique singulière: cette appartenance est assumée consciemment par le philosophe lui-même. Sa théorie de l'Histoire et les principes de sa rhétorique l'obligent, en effet, à reconnaître sa "situation" dans le siècle, à parler d'un lieu particulier à un auditoire aussi particulier. Cette adhésion à son temps est d'autant plus nécessaire que sa pensée est animée par une vocation essentiellement pratique: ce sont ces hommes-ci les hommes de mon temps, qu'il faut essayer de réformer pour les sauver. Et c'est pourtant au moment même qu'on reconnait cette adhésion au présent et à la situation historique qu'on découvre qu'il n'y est jamais comme le poisson dans l'eau et qu'il les fuit déjà et toujours vers un autre temps et qu'il parle "du dehors". S'adressant ainsi à un "lecteur futur", Rousseau semble attendre les re-centrements de son discours par un commentaire futur: les "jours nouveaux" et une pensée encore à naître sont comme que preparés dans l'intimité de l'oeuvre.

Mais le parallélisme avec Leibniz n'est qu'apparent: la positivité de la récurrence dans l'histoire de la lecture de Leibniz est garantie par son insertion dans l'histoire de la science et par le caractère toujours complémentaire des commentaires. Si les differentes manières de centrer le discours de Leibniz sont complémentaires c'est, indique M. Serres, parce que ce discours est, en lui-même, sous-tendu par une structure "à plusieurs entrées". Chez Rousseau, au contraire, les différentes lectures ne conspirent jamais et peuvent même suggérer quelque chose comme une "excentricité" essentielle de l'oeuvre. La divergence des lectures pourrait renvoyer, au contraire du cas de Leibniz, à une inconsistance radicale ou à un manque de prégnance dans la structuration du discours. La multiplicité des lectures rivales pourrait, en effet, être le signe d' une indétermination dans l'ordre du discours en lui-même et, dans ce cas, la récurrence perd sa positivité et l'idée d'illusion rétrograde réapparaît dans son sens négatif.

On a toujours parlé, en effet, d'une "excentricité" de Rousseau, et lui le premier. L' excentricité a alors un sens psychologique, que n'est pas son seul sens, ni le plus profond. Des mots 
comme bizarre lui viennent souvent à la plume quand il fait son propre portrait. Ce que ce langage signifie c'est exactement une sorte d'absence de centre, un mouvement constant entre des extrêmes. Déjà en 1749, dans le Persifleur, comme dans tous les écrits autobiographiques postérieurs, le thème est présent:

"Quant Boileau a dit de l'homme en général qu'il changeait du blanc au noir, il a croqué mon portrait en deux mots (...) Rien n'est si dissemblable à moi que moi-même, c'est pourquoi il seroit inutile de tenter de me définir autrcment que par cette variété singulière (...) Quelquefois je suis un dur et féroce misantrope, en d'autres momens, j'entre en extase au milieu des charmes de la société et des délices de l'amour (...) En un mot, un protée, un Cameléon, une femme sont des êtres moins changeans que moi" (46).

Dans l'alégresse du texte on reconnait la même structure qui plus tard va animer des textes plus pathétiques. Marcel Raymond a bien analysé ces mouvements comme des mouvements pendulaires autour d'un centre qui n'est jamais atteint dans le repos, autor duquel l'existence ne peut pas se resserrer, se recentrer pour entrer en possession d'elle-même. Tous ces changements successifs on pourrait parler d'un "terrorisme" de l'instant que ne cesse de briser la continuité du temps et de l'ego —, dit M. Raymond:..

“... se réduisent souvent à un rythme pendulaire. On lit dans les Confessions, au sujet du drame de l'Ermitage: "des lors, mon âme en branle n'a plus fait que passer par la ligne de repos, et ses oscilations toujours renouvelées ne lui ont jamais permis d'y rester". Durant des mois d'extravagance, en effet, sa conduite à l'égard de ses amis, Mme. d'Epinay, Grimm, cst marquée, avant la rupture, par une suite d'oscilations, ou mieux, de renversements du pour au contre allant de l'amitié la plus vive, avec tous les aveux et les larmes de la tendresse, à la fureur et aux gestes de la sensibilité la plus agressive" (47).

Cette excentricité est aussi le trait essentiel par lequel Rousseau apparaît à ses contemporains et qui les amène à osciler, eux-mêmes, entre les hypothèses extrêmes de la méchanceté et de la folie. Il est l'"ours" qui choisit la solitude, quand il peut vivre à Paris; tous ses choix, enfin, tout le style de son existence quotidienne lui désignent comme quelqu'un qui échappe à la norme et offre le flanc aux interprétations les plus perfides.

Mais cette excentricité personelle est moins importante que l'excentricité de son oeuvre elle-même, dont on peut dire qu'elle

(46) O. C., I, p. 1108.

(47) M. Raymond, J.-J. Rousseau, la quête de soi et la rêverie, J. Corti, p. 23. 
dérive. La "réforme morale", la décision de devenir "autre" et d'abandonner le monde - la première occasion de conflit avec les philosophes - n'est elle pas motivée par la pensée qui est née de l'illumination de Vincennes? Pensée excentrique, donc, car elle brouille les lignes qui partagent et structurent le champ idéologique de son temps. Qu'on soit dévot ou qu'on soit philosophe, on a toujours un lieu précis dans ce champ: n'importe quel discours est compréhens.ble, à condition de respecter cette opposition fondamentale. Mais, quoi! un philosophe dévot! La clarté rassurante de l'opposition est mise en péril et, avec elle, toutes les évidences courants. On ne voit plus ce centre qui sépare une "gauche" d'une "droite", ce lieu de partage où s'opposent et où se font la guerre les seules options théoriques apparement possibles. Quel projet plus insensé que celui de la Nouvelle Héloïse, d'établir une "coexistence pacifique" entre l'athée et le croyant? La haine de Voltaire y trouve son fondement: Rousseau lui apparaît comme traître, comme celui qui masque le véritable combat de la Raison.

Le fait est donc que l'oeuvre de Rousseau ne reproduit pas, dans son espace intérieur, la ligne de partage qui divise le champ idéologique de son siècle. Ou, autrement, la surface même de ses écrits indique que le conflit essentiel n'est pas celui qui apparâ̂t comme tel à la conscience de ses contemporains .

Comme une sorte de monade infidèle, qui ne reproduit pas exactement dans son intimité la carte du monde extérieur, miroir déformant qui concilie des inconciliables, elle est vue comme foncièrement contradictoire. Ce miroir fracturé ne nous restitue pas la vérité du monde et, dans l'éclat éblouissant de l'image il cache les distorsions de cette image, elle-même felée et contradictoire. L'excentricité, intériorisée et devenue la loi de l'oeuvre, devient contradiction et son déguisement devient sophistiquerie. L'éclat du style cache les contradictions (voire la folie) de la pensée, comme le trompe-l'oeil de l'image miroitée cache l'infidélité du portrait. L'erreur, l'infidélité est donc le résultat d'un manque de centre: la contradiction - mouvement pendulaire qui conduit du oui au non, qui passe de l'affirmation à la négation - est le fruit d'un décentrement essentiel à l'oeuvre, d'une secrète maladie qui reproduit au niveau du discours la "folie" de son auteur.

Ce manque de centre, cette gravité déplacée vers la dehors et donc instable, n'est seulement la thèse des adversaires de Rousseau. L'instablité de son discours est décrit aussi, sans la moindre intention polémique, par exemple, dans deux essais ré- 
cents - Sur le "Contrat Social" de Louis Althusser et Gravité de Rousseau de Alain Grosrichard (48).

Le point de départ de l'analyse de Alain Grosrichard est I'aftirmation que la loi de l'oeuvre de Rousseau réside dans la ligne qui la sépare de son dehors. Le coeur même de l'oeuvre, son intimité la plus profonde et enfouie, est ouverture sur l'extériorité qui la nie et qui est niée par el'e. Cette tension entre le dedans et le dehors de l'oeuvre - ce lieu de rupture - est la répetition dans le niveau du discours d'une expérience muette qui la precède: "Il n'y aurait pas d'oeuvre si, au départ, il n'y avait pas eu dou'eur, contradictions, déchirement du sujet" (49). C'est dire, en plus d'un sens, que l'oeuvre est encore excentrique: d'abord parce qu'elle naît de quelque chose qui n'est pas de l'ordre de l'oeuvre et surtout parce qu'elle est une réponse à ce qui est l'autre de l'oeuvre: "L'oeuvre de Rousseau est profondément une oeuvre de réaction. Elle ne faît, ne se constitue, ne se développe en système que de se provoquer elle-même" $(50)$.

On ne voit p'us maintenant, dans cette excentricité, la preuve d'une mauvaise foi et d'une méchanceté, mais elle peut donner l' occasion de l'affirmation d'une manque au coeur de l'organisation conceptuelle de l'oeuvre. Si l'oeuvre oscile autour d'un centre qui lui est extérieur, il se peut qu'il soit impossible de lui trouver une signification univoque: si l'on ne parle plus de sophistiquerie, on peut toujours parler d'une indécidabilité du sens. Si l'on peut attribuer plusiers sens à l'oeuvre, ce n'est pas par la faute de la lecture ou parce que les interprétations sont complémentaires, mais par faute de l'oeuvre elle-même qui ne cesse de tourner autour d'elle-même et qui ne peut pas se fixer dans un sens premier ou unique. L. Althusser, examinant le fonctionnement logique de cet "objet philosophique" qu'est le Contrat Social croit trouver la loi de sa structure dans un "décalage" interne qui, produisant successivement d'autres décalages, permet de poser et de résoudre un problème, mais seulement à condition de cacher la malice de ces décalages. Le décalage a son origine dans la définiton même du contrat et dans la nature "particulière" de ce contrat: au contraire des autres contrats, ici une des "Parties prenantes" ne précède pas le contrat; elle est, au contraire, produite par le contrat qu'elle est supposée prendre. Il n'y a pas, à proprement parler, un sophisme, mais un vide théorique, le refoulement d'un hyatus et d'un désequilibre logique qui ne saurait être dépassé que par

(48) Tous deux publiés in "L'impensé de Jean-Jacques Rousseau", Cahiers pour 'Analyse, $\mathrm{n}^{\mathrm{2}} 8$.

(49) Cahiers pour l'Analyse, $\mathrm{n}^{2} 8$, p. 43.

(50) Ibid. 
une autre forme de pensée. Dans son essai, le projet d'Althusser est de construire rigoureusement l'espace ouvert par ce hyatus et de donner ainsi le fondement de l'errance de la pensée de Rousseau, ainsi que des plusieurs "lectures" dont elle est susceptible:

"...rendre intelligible la possibilité de plusieurs "lectures" du Contrat Social, et les interprétations subséquents (kantienne, hegelienne, etc.). Ces interprétations ne nous apparaitront comme simplement arbitraires et tendencieuses, mais comme fondées, en leur possibilité, dans le texte même de Rousseau: très précisément dans le "jeu" permis par l'"espace" des Décalages théoriques constitutifs de la théorie de Rousseau. Ces interprétations pourront servir à leur tour d'indice et de preuve de l'existence nécessaire de ces Décalages" (51).

\section{V \\ Vers le centre rhétorique}

"Ce que les anciens ont fait avec l'éloquence est prodigieux..." (52).

Rousseau.

"... tandis que notre civilisation, caracterisée par son extrême ingéniosité dans les techniques destinées à agir sur les choses, a complètement oublié la théorie de l'argumentation, de l'action sur les esprits au moyen du discours, celle-ci était considerée par les Grecs, sous

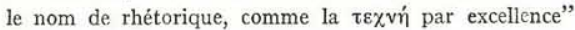
(53).

Ch. Perelman et Olbrechts-Tyteca.

L'oeuvre de Rousseau n'aurait-elle donc point de centre? Le lecteur ne pourrait-il pas accéder à ce point géometral dont parlait Leibniz, ce lieu unique d'où le spectateur serait capable de voir l'unité secrète de l'objet de son regard? Toute une tradition refuse l'existence de ce géometral. Et Althusser fait de cette absence la théorie exhaustive: cette lacune donne la loi de l'oeuvre, dont tout le mouvement n'est guidé que par la nécessité de la cacher. On ne

(51) Ibid., p. 6.

(52) O. C., IV, p.

(53) Ch. Perelman et L. Olbrechts-Tyteca, Traité de l'Argumentation, Presses Universitaires de France, V. I, pp. 11-2. 
peut que tourner autour de cette ville qui n'a pas de dedans. Il ne s'agit pas, comme disait Hegel, de l'architecture "symbolique" des Egyptiens (ainsi que du langage abstrait des mathématiques) d'un temple vide qui n'abrite que l'absence de Dieu; il s'agit plutôt d'un faux temple, qui se défait à mesure que le spectateur erre autour de lui. On n'est pas, finalement, très loin de la conception polémique de l'oeuvre de Rousseau; cette construction laborieuse ("penser fût toujours pour moi une occupation pénible et sans charme") n'est finalement qu'un château de cartes: comme un souffle imprudent, la lecture attentive, montrant le néant des articulations logiques, la fait sombrer dans son vide théorique, ou dans le trop plein de la littérature (54).

On avait cru que, au long de l'histoire de la lecture de Rousseau, on avait fini par supprimer l'abîme entre théorie et littérature. Le voilà qui ressurgit avec une profondeur accrue. S'il y a une lecture qui unifie l'oeuvre, elle ne le fait qu'en la réduisant à l'expression d'une existence. $\mathrm{Si}$, au contraire, la lecture essaie de reconstituer l'articulation logique de l'oeuvre, l'opposition entre la théorie et son autre éclate à l'intérieur même de la théorie et la brise du dedans. Expression ou théorie? Littérature ou concept? Les interprétations récentes nous liberé de toute une série de préjugés et ont rendu possible la lecture de Rousseau: mais devant le texte ainsi liberé nous retrouvons les perplexités de toujours. Le problème de l'unité de la pensée de Rousseau reste entier.

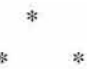

L'unité de l'oeuvre n'est pas, en effet, la même chose que l'unité de la pensée. Si l'on peut montrer que l'oeuvre oeuvre est unitaire dans la mesure où elle exprime une même existence, dans la mesure où dans les livres, théoriques ou non, ou retrouve les même thèmes et les mêmes obsessions, on ne peut pas considérer cette convergence thématique comme preuve de cohérence théorique. On donne vie aux textes, on dramatise les concepts, mais pour trouver leur origine ou leur fondement, en tout cas leur vérité, dans les contradictions d'une existence. Voyons, par exemple, comment J. Starobinski (55) rend compte de l'ambiguite de l'idée de réflexion dans la pensée de Rousseau:

D'un côté, la réflexion est la condition et l'occasion de la déchéance, elle ouvre entre le désir et son objet la distance infinie du travail, du temps, des médiations et de l'objectivité. Elle secrète toutes les idoles de la médiation - qui acquièrent un poids auto-

(54) L. Althusser, op. cit., dernier paragraphe.

(55) J. Starobinski, L'Oeil Vivant, Gallimard, 1961, pp. 83-188. 
nome et cachent la fin qu'elles devraient rendre accessible - comme l'argent, la propriété et le langage: "... ce sont les liens qui enchainent à chaque acte la série de ses conséquences" (56). La réflexion est donc à l'origine de tout ce que Rousseau refuse, dans ses pulsions les p'us spontanées ainsi que dans les concepts qu'il produit; elle est même le grand danger que le "philosophe" doit condamner, en condamnant la Philosophie. Mais comment condamner la réflexion, sans réflechir? L'existence "pré-réflexive" coule calmement comme la rivière, elle est naturelle et, dans son innocence, elle ignore les dangers et les péchés de la réflexion. Découvrir, par la réflexion, les périls de la réflexion, c'est presque les avoir dépassés; mais ce n'est pas encore les avoir éliminés: c'est encore s'exposer et subir le risque: "C'est peu de dire qu'en combattant la vie déchue il devient son captif: s'il la supporte avec impatience, comme une fatalité imméritée, il sait aussi qu'elle est la seule voie possible, et que, pour en sortir, il faut l'avoir traversée" (57). On s'expose aux périls de la réflexion parce qu'il n'est plus possible d'être spontané ou naturel et le comble de la réflexion - la réflexion sur la réflexion - peut devenir son contraire. Mais Starobinski montre bien qu'il ne s'agit pas d'un schéma dialectique: l'issue n'est pas nécessaire et on peut toujours retomber dans la barbarie de la réflexion. La négation de la négation n'est pas nécessairement une affirmation et le schéma renvoie plutôt à une ambiguité qu'à une dialectique. A la fin on vérifie que ces contradictions sont indépassables, qu'elles sont des contradictions toujours vivantes, car ce sont des contradictions surtout vécues.

Ce sont bien des contradictions vécues qui servent de fil conducteur à la belle analyse faite par Starobinski: tout s'explique à la lumière de l'expérience originaire du désir coupable et de 'l'effort pour séparer le désir de sa culpabilité. La stratégie du désir commande la vie comme l'oeuvre:

"En fait tout se passe comme si le monde était trop étroit pour la présence simultanée de la conscience désirante, de l'objet convoité et du témoin sévère. Leur affrontement provoque un malaise intolérable. Il faut que l'un des trois se dissimule, se transforme ou disparaisse" (58).

Mais l'unité n'est alors que le rêve que cette existence poursuit, le projet toujours irréalisable de devenir un et d'expulser du monde et de soi tout conflit et toute contradiction, le projet, enfin, de devenir Dieu. Et c"est pourquoi Starobinski refuse à Rousseau le droit de dire, à la fin de sa vie, qu'il a atteint finalement l'unité

\footnotetext{
(56) Ibid., p. 154 .

(57) Ibid.

(58) Ibid., p. 104.
} 
et le calme de la conscience apaisée: "il est encore à mi-chemin" (59). Si l'unité de l'oeuvre est l'unité de l'existence, elle est toujours future et impossible, unité désirée au sein d'une existence toujours contradictoire:

"Il a beau vouloir se convaincre qu'il est parvenu au terme: est-il en mesure de s'en assurer? Le temps de la division et du devoir réflexif est-il vraiment révolu? La réflexion a-t-elle abouti? Rousseau voudrait le croire mais il n'en est pas certain lui-même. D'où les accès d'angoisse qui l'assaillent jusque dans l'expression des certitudes désirées: "Ah! me disois-je alors dans des serrements de coeur prêts à m'éttouffer, qui me garantira du désespoir?" (60).

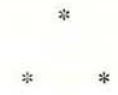

D' autre part, comment chercher la vérité de l'oeuvre de Rousseau dans l'unité d'une téorie? Pourquoi, en effet, essayer de monter l'appareil d'un "système", si Rousseau lui-même déclare vouloir nous libérer de cet "effrayant appareil de philosophie? L'impossibilité d'une telle entreprise semble donc être prouvée par des déclarations explicites du philosophe. Mais, plus encore, elle semble l'être aussi par le style même de ses écrits, par le mouvement même de sa pensée telle qu'elie s'objective dans la structure de son discours. On pourrait, en effet, trouver un "système", une "doctrine" plus ou moins cohérente, à la base de tous ses écrits, mais cette doctrine ne serait, alors, que le résidu dogmatique de sa pensée. Nous n'opposons pas ici, à la manière de Bergson, l'intuition profonde à son expression linguistique et conceptuelle - expression qui livre l'intuition, mais qui la cache aussi et la déforme -, mais la logique d'une pensée - telle qu'elle s'incarne dans la structure même du texte - aux thèses ou aux dogmes auxquels elle aboutit, L"interêt de l'analyse de Althusser - et c'est en cela justement quil différe des commentateurs qui dénoncent l'incohérence de Rousseau - est de montrer l'inconsistance de la théorie, non pas dans une contradiction entre des thèses différentes, mais dans les procedès logiques qui lui permettent d'engendrer ces thèses. S'il est vrai que c'est dans le mouvement même de l'argumentation que se trouve l'inconsistance, toutes les interprétations rivales de Rousseau sont justifiées, mais en même tamps frappées de nullité. Le vide théorique qui s'insinue dans les fissures de l'argumentation serait quelque chose comme un miroir où toutes les théories (kantisme,

(59) Ibid., p. 187.

(60) Ibid., pp. 187-8. 
hegelianisme, existentialisme) pourraient reconnaitre leur propre image.

Reprenons, toutefois, le problème de la structure du discours de Rousseau. Une chose est certaine, et Rousseau est le premier à nous le dire et à nous fournir les raisons: on ne peut pas trouver dans ses textes un ordre linéaire de raisons. Et cela parce que un tel ordre correspond au modèle le plus évident du discours scientifique, alors que Rousseau n'attribue jamais à son discours un tel statut. La science au sens fort du mot, comme connaissance qui a trouvé um fundamentum absolutum à partir duquel elle peut procéder apodictiquement, ne lui semble pas être à la portée de l'entendement fini des hommes. Le savoir fondé dans l'Absolu est pour lui l'apanage de l'entendement infini de Dieu; mais il est. alors le fait d'une vision instantanée de la totalité du réel. Le discours, symbole de la finitude, ne peut pas reproduire l'Ordre de la Nature.

"Je fis voir que la source de nos erreurs sur ce point vient de ce que nous confondons nos vaines et trompeuses connaissances avec la souveraine intelligence qui voit d'un coup d'oeil la vérité de toutes choses. La science prise d'une manière abstraite mérite toute notre admiration. La folle science des hommes n'est digne que de risée et de mépris" (61).

Chez Descartes, la véracité de Dieu, une fois démontrée son existence, donne à la série des évidences de "ma" science (j'existe, je suis une chose qui pense, l'âme est plus facile à connaitre que le corps) une portée ontologique: l'ordre de la connaissance finit par rejoindre l'ordre de l"être. Par la médiation de Dieu, l'entendement fini peut reproduire, dans l'ordre de ses évidences, l'Ordre de la Nature. Chez Rousseau, l'Ordre de la Nature est toujours postulé, mais il ne peut pas être reconstruit méthodiquement par la connaissance ou, au moins, apodictiquement. L'Ordre n'est pas évidemment un noumène inaccessib'e: il peut se dévoiler dans des expériences privilégiées. Dans ces moments, le sentiment de l'existence semble même abolir, l' abîme qui sépare le fini de l'infini.

Mais seul le sentiment peut ainsi nous approcher de l'infini et la connaissance ne peut jamais trouver le fundamentum absolutum qui lui permettrait de se constituer en système. L' enchainement du Savoir d'après un ordre linéaire de raisons est exclu comme possibilité dans l'horizon de la pensée de Rousseau.

Il $y$ a, il est vrai, des textes où Rousseau non seulement affirme l'unité de sa pensée ("J'ai écrit sur divers sujets, mais toujours

(61) O. C., II, p. 965. 
dans les mêmes principes ..."), mais où il indique en plus quelque chose comme un "ordre de raisons" qui fonderait cette unité au delà du disparate des matières traitées. C'est ainsi que le Français dit dans les Dialogues:

"J'avais senti dès ma première lecture que ces écrits marchaient dans un certain ordre qu'il fallait trouver pour suivre la chaîne de leur contenu. J'avois cru voir que cet ordre était rétrograde à celui de leur publication, et que l'Auteur, remontant de principes en principes n'avait atteint les premiers que dans ses derniers écrits. Il fallait donc pour marcher par synthèse commencer par ceux-ci, et c'est ce que je fis en m'attachant d'abord à l'Emile par lequel il a fini"' (62).

Rousseau semble opposer ici, comme Descartes, un ordre des raisons à un ordre des matières. Et, en effet, ce texte dénonce le même aveuglement que $M$. Gueroult voit dans la plupart des interprètes de Descartes, qui "ne voient en lui qu'une succession biographique et non un enchainement rationnel, et ne font en réalité qu'observer la suite simplement chronologique des matières" (63). Mais quel est cet ordre qu'il faut suivre pour saisir la "chaine" des contenus? Il s'agit d'un ordre synthétique: un ordre rétrograde à celui de la publication ou de l'énonciation, on pourrait dire, parce que l'ordre chronologique est celui de l'analyse, de la régression vers des principes chaque fois plus universels. C'est comme si Rousseau nous disait que seul l'ordre synthétique pouvait rendre visible l'unité de sa pensée. Nous sommes donc déjà très loin de Descartes où le système n'est vraimente fondé que par l'analyse, tandis que la synthèse n'est qu'une forme d'exposition d'un savoir validé et construit par l'analyse. Mais la véritable différence est ailleurs: l'ordre n'est pas chez Rousseau, constitutif comme il l'est chez Descartes, il ne fournit pas la règle de la génération des propositions. Au fond, Rousseau dit simplement que sa pensée est aussi susceptible d'une exposition synthètique: cette exposition pourrait rendre visible sa cohérence, mais ne saurait la fonder ni démontrer sa vérité. En effet, quelques paragraphes auparavant, le Français marquait nettement cette limitation, en parlant d'un "système lié qui pouvait n'être pas vrai, mais qui n'offroit rien de contradictoire". L'ordre n'est pas donc le garant de la vérité, il ne peut garantir que la non-contradiction. Mais, surtout, nous nous trouvons encore une fois devant une unité idéale et future, qui ne nous apprend rien - sinon négativement — sur la structure de son discours. Et d'ailleurs c'est moins l'attention à l'ordre

(62) Dialogues, Bibliothèque de Cluny, p. 273.

(63) M. Guéroult, Descartes selon l'ordre des raisons, t. I, p. 13. 
qu'une "bonne disposition" qui permet de dissiper l'illusion des contradictions:

"Je ne tardais pas à sentir en lisant ces livres qu'on m'avoit trompé sur leur contenu, et que ce qu'on m'avait donné pour des fausses déclamations, ornées de beau langage, mais décousues et pleines de contradictions, étoient des choses profondement pensées et formant un système lié qui pouvait n'être pas vrai, mais qui n'offroit rien de contradictoire. Pour juger du vrai but de ces livres, je ne m'attachai pas à éplucher çà et là quelques phrases éparses et séparées, mais me consultant moi-même et durant ces lectures et en les achevant, j'examinois comme vous l'aviez désiré, dans quelles dispositions d'âme elles me mettoient et me laissoient, jugeant comme vous que c'étoit le meilleur moyen de pénétrer celle où étoit l'Auteur en les écrivant, et l'effet qu'il s'étoit proposé de produire" (64).

La découverte du bon ordre de lecture passe ainsi par la bonne volonté: la lumière qui anime le discours peut trouver un écho dans un autre coeur et faire qu'il soit aussi illuminé du dedans: mais elle ne peut pas s'imposer du dehors, dans l'espace de l'objectivité, par la coercition de la cience.

Faut-il donc renoncer à trouver l'ordre qui commande l'oeuvre de Rousseau et reconnaître dans son centre quelque chose comme un vide théorique? Ou bien faut-il dénoncer dans la thèse du désordre et de la contradiction l'effet d'une lecture qui part d'une idée étroite de l'ordre et qui le cherche là où il ne se trouve pas? Nous savons déjà, en effet, que l'ordre linéaire et irréversible des raisons n'est pas le seul modèle de discours philosophique cohérent. L'exemple de Leibniz, tel qu'il a été interprété par M. Serres, est ici pertinent: l'impossibilité de reconstituer un système selon le modèle cartésien ne signifie pas nécessairement que ce système n'est pas ordonné:

"Poser cette question c'est être victime de ce que le XVIIe. siècle entendait, croit-on, massivement, par le terme rigueur où enscignait en général sous le titre "méthode". A ce sujet, les leçons ordinaires de Descartes ou de Spinoza portent l'examen à une confusion qui consiste à identifier pensée cohérente (rigoureuse, systèmatique) et pensée déductive, alors que la première contient la dernière comme cas particulier" (65).

(64) O. C., I, p. 930.

(65) M. Serres, Le système de Leibniz, Presses Universitaires de France. 
Mais, de même que l'identification entre ordre et déduction est une limitation de l'idée moderne d'ordre, on pourrait aussi suggérer que le modèle mathématique n'est pas le seul à pouvoir donner un sens à l'idée d'ordre et à servir de guide au discours philosophique. Le compromis entre mathesis et ordre est bien un compromis qui a été noué par la pensée moderne. Mais, est-ce qu'il ri'y a pas, chez Rousseau, justement un refus de l'idée moderne de la philosophie, et l'exigence d'un retour à un autre style de pensée? Rousseau pourrait bien dire que l'ordre mathématique,qu'il soit déductif ou non, n'est qu'un cas particulier de l'ordre qui se donne aussi par une expérience esthétique ou morale. Il faut surtout insister sur l'étroit liaison entre la limitation de la mathésis et l'éloge de l'antiquité. Un commentateur récent observe:

"Un exact géomètre pourra être fort injuste, pour peu que lui manque cet amour de la vertu qui incline vers le bien, comme le fort bon théologien pourra tout ignorer de la foi qui anime le Vicaire savoyard. Rousseau retrouve par là une tradition lointaine, occultée par la formule du rationalisme cartésien: "Il suffit de bien juger pour bien faire". Si le dialecticien philosophe du livre VI de $L a R e ́-$ publique définit les lignes proportionnelles qui lui permettront d'atteindre le lieu où se manifeste l'idée de Bien, encore faut-il se souvenir, du Phèdre à la République, qu'il doit être un dialecticien amoureux, tourné, tendu du côté du Bien par l'Eros, qui de l'amour des belles formes à l'amour de l'idée du Bien, stimule le courage, oriente la quête philosophique. Il ne s'agit pas d'être ignorant, il s'agit de savoir être ignorant: il serait absurde de vouloir l'être, mais il est possible d'atteindre cette docte ignorance, que seule assure la coïncidence de la vérité et de la justice. Socrate est présent à la pensée de Rousseau, comme lui est présent le Christ: il réunit dans le même hommage "le sage hébreu et le sage grec" (66).

Le refus de la modernité et l'éloge de l'antiquité pourrait bien être plus qu'un thème parmi d'autres dans la pensée de Rousseau. Dans ce mouvement régressif, dans cette découverte progressive de principes chaque fois plus universels, ne pourrions-nous reconnaitre un écho lointain de la méthode de Socrate ou de Platon, beaucoup plus qu'une répétition des procédés courants de la Raison Moderne? On a déjà beaucoup parlé, et avec justesse, du p'atonisme de Rousseau: les Dialogues nous parlent de l'autre monde, les $R \hat{e}$ veries définissent la philosophie et la sagesse comme préparation à la mort, le Contrat Social définit les conditions de la Cité juste, etc ... Mais, surtout, l'idée d'Ordre apparaît comme modèle au 
sens platonicien: elle est en même temps le Beau, le Vrai et le Bon qu'il faut essayer d'imiter ou de reconstituer au sein de ce monde de ténèbres et de désordre. Mais ce platonisme, il faut le reconnaître, est essentiellement limité et la régression vers le principe ne s'accomplit pas, comme dans la dialectique, par la certitude de l'epistemê: le modèle ou l'archétype ne se donne jamais dans une noesis pure, et on n'abandonne jamais le monde du mixte. C'est ce qui apparaît, par exemple, dans l'analyse que Patrick Hochart fait des rapports entre le modèle et son simulacre à propos du système de renvoi qui s'établit dans les textes de Rousseau entre la Société Civile, la Société Universelle et les sociétés "pathologiques" commes celles des brigands:

"Ainsi le simulacre est ennemi du modèle dans la mesure où il est fondamentalement autre et où il ne se constitue que dans et par son absence. Simulacre et modèle sont donc incompatibles dans leur présence comme la société civile et la société générale et le simulacre défigure, déshonore le modèle. Et pourtant le simulacre possède un lien de filiation irréductible qui l'attache au modèle dont il procède et à ce titre il est ce qui sauvegarde le modèle et en le détruisant on se couperait définitivement de toute vois d'accès au modèle, comme en détruisant la société civile on détruirait toute société. Le simulacre est ainsi pensé à mi-chemin du modèle et du leurre, qui comme "faux simulacre" ou hyprocrisie aurait totalement rompu avec le modèle tout en paraissant le recouvrir parfaitement, et il tient toujours des deux à la fois. Mais le simulacre ne surgit pas innocemment pour remplacer le modèle originaire qui lui préexisterait et viendrait inexplicablement à s'absenter, il est ce qui détruit le modèle et le même mouvement abolit le modèle et constitue le simulacre. Ainsi la lutte entre le simulacre et le modèle est originaire (simul); le premier a toujours déjà commencé à défigurer le second et le second à être simulé par le premier; en ce sens le modèle renvoie tout autant au simulacre que le simulacre au modèle. C'est pourquoi les deux termes en référence a quoi le simulacre a été déterminé (le modèle et le leurre) sont toujour déjà et toujours encore travaillés par lui et ne peuvent recevoir une détermination pure" (67).

A partir de cette analyse, on pourrait arriver à la conclusion qu'il n'y a pas seulement une limitation du platonisme chez Rousseau, mais déjà un renversement. En effet, si la puissance propre de la dialectique, ce qui définit son style spécifique, est moins de "diviser un genre en espèces contraires pour subsumer la chose

(67) Patrick Hochart, "Droit naturel et simulacre", in Cahiers pour l'Analyse, n 8, p. 81 . 
cherchée sous l'espèce adéquate" (68) - car la division ne serait alors qu'un syllogisme impuissant - que de "sélectionner des lignées, distinguer des prétendants, distinguer le pur et l'impur, l'authentique et l'inauthentique" (69), cette complicité entre le modèle et le simulacre est déjà la mort de la dialectique. L'ambiguité du simulacre qui cache le modèle en occupant sa place vacante, mais qui renvoie tout de même au modè'e disparu et en fournit un "supplément", est aussi à l'origine de l'ambiguité du jugement de la valeur des sciences et des arts: car "Elles détruisent la vertu, mais elles en 'aissent le simulacre public qui est toujours une belle chose" (70). L'impossibilité d'assimiler le simulacre au leurre, en dépit de la déformation manifeste qui en résulte pour le modè e, est nettement affirmée par la définition que Rousseau en donne dans la préface du Narcisse:

"Ce simulacre est une certaine douceur des moeurs qui supplée quelquefois à leur purété, une certaine apparence d'ordre qui prévient l'horrible confusion, une certaine admiration des belles choses qui empêche les bonnes de tomber toutefois dans l'oubli. C'est le vice qui prend le masque de la vertu, non comme l'hypocrisie pour tromper et trahir, mais pour s'ôter sous cette aimable et sacrée effigie l'horreur qu'il a de lui-même quand il se voit à découvert" (71).

Si la puissance de la dialectique est bien, comme le dit G. Deleuze, de pouvoir toujours séparer les copies-icônes des simulacres-phantasmes, l'inspiration platonicienne de Rousseau et tous les thèmes qu'il hérite de Platon sont neutralisés et on ne peut pas dire que son discours est de l'ordre de la dialectique.

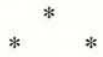

Mais il ne faut pas penser cette limitation de la dialectique de manière puremente négative: il faut s'interroger sur le genre de discours qu'elle rend possible. Chez Aristote, par exemple, cette limitation est condition de la ibération de la science comme discours autonome, qui n'a plus besoin d'un fondement anhypothétique pour assurer sa propre scientificité. Chez Isocrate, d'autre part, te discours politique n'a pas besoin d'un fondement absolu dans l'epistemê pour être vrai, juste et utile: c'est la rhétorique, alors, qui acquiert son autonomie et qui n'a pas besoin de la vision immédiate de l'Idée pour fonder sa vérité. Chez Platon, la rhétorique, coupée de la science de la dialectique n'était que bavardage; chez

(68) G. Deleuze, La logique du sens, Minuit.

(69) Ibid.

(70) O. C., II, p. 972.

(71) Ibid. 
Isocrate, c'est la spéculation qui, coupée des problèmes urgents de la Cité, devient pur jeu de mots: c'est ainsi qu'il semble adresser à Platon les mots suivants:

"Qu'ils cherchent la vérité, qu'ils forment leurs disciples à la pratique de notre vie politique, qu'ils les entrainent pour leur donner l'expérience de cette vie, avec la conviction dans l'âme qu'il vaut mieux apporter sur des sujets utiles une opinion raisonnable que sur des inutilités des connaissances exactes: qu'il vaut beaucoup mieux marquer un avantage discret sur un grand sujet, plutôt qu'une écrasante supériorité à l'occasion de médiocres, sans utilité pour la vie humaine" (72).

$\mathrm{Ne}$ reconnaissons-nous pas, dans cette mise en accusation de la vaine rigueur des philosophes, la critique adressée par Roussea!ı "à ces enfants qu'on appelle des phi'osophes", qui s'attardent dans la discussion de problèmes insolubles et laissent de côté les questions importantes de la morale et de la politique? Comme l'indique $\mathrm{P}$. Burgelin à propos de Rousseau (73), on ne peut pas parler d'un "utilitarisme étroit", mais on peut certainement parler d'une subordination de la vérité théorique à la vérité pratique. La critique de la Philosophie a la même inspiration chez Rousseau et chez Isocrate: le philosophe est le raisonneur, la victime d'une folle hybris qui veut contenir dans les faibles mailes de son discours la totalité du réel. $\mathrm{Au}$ philosophe, Isocrate comme Rousseau opposent la finitude du Savoir humain et l'impossibilité de décider avec certitude entre les hypothèses rivales, de découvrir parmi tous les systèmes du monde quel est le vrai. A une vaine préocupation théorique, toujours condamnée à l'indécidabilité, les deux critiques de la Philosophie oposent la préocupation plus sérieuse de la morale et de la politique; préocupation avec des probèmes qui sont susceptibles de solution au niveau d'une orthodoxie, d'une opinion droite et raisonnable et qui n'a besoin de chercher dans une epistemê quelconque sa vérité. C'est, en effet, une sorte de foi ou de bonne foi qui occupe le lieu laissé vide par une science désormais impossible: et avec cette bonne foi, c'est la rhétorique qui acquiert la dignité de discours vrai, même si elle n'aspire pas à une vérité absolue. Cette vérité dont elle est capable - et qui lui donne sa dignité - n'est plus evidemment la vérité eternelle d'une Raison intuitive, mais une vérité locale et éphémère qui est pourtant la seule sur laquelle peut compter la décision pratique et qui seu'e peut répondre à l'urgence de la vie morale et politique. Il s'agit d'une conception plus humble de la vérité, mais c'est justement cette humilité qui est à la hauteur des graves décisions qu'elle rend possibles. C'est, au fond, l'idée

(72) Isocrate, L'éloge d'Hélène, Belles Lettres, § 5, t. I, pp. 164-5.

(73) P. Burgelin, op. cit., pp. 103-12. 
du kairos qui commande cette conception du discours et de la vérité - le kairos est cet instant éphémère qui éclate dans le temps urgent et rapide où les cités justes peuvent se précipiter dans la corruption et dans l'injustice. Pour Rousseau, d'ailleurs, cette urgence est aussi présente dans l'expérience la plus solitaire de l'exis. tence, au dehors des murs de la cité, et la morale du solitaire est aussi hantée par ce temps qui passe et qui peut rendre l'effort de réforme morale trop tardif; le salut des âmes, comme celui des collectivités, s'expose au risque avec le temps qui passe. C'est ainsi que dans les Rêveries la méditation morale se fait à la charnière entre l'anticipation de la mort et la réf'exion sur le temps perdu: "Est-il temps au moment qu'il faut mourir d'apprendre comment on auroit dû vivre?" (74). Et chez Rousseau cette urgence est d'autant plus grande, que l'humanité ne peut pas rétrograder, de même que le vieux ne peut pas redevenir jeune. La sagesse serait presque toujours rétrospective: savoir ce qu'on aurait dû faire et que malheureusement on n'a pas fait. Le rhéteur ne prend la parole que pour manifester le commencement de la corruption, pour dénoncer le mal qui commence à étendre son domaine, il parle dans ce temps bref qui sépare l'émergence d'un problème du moment où toute so'ution deviendra impossible. Pour caractériser cette conception de la praxis, on pourrait dire que, pour Rosseau, l'humanité ne se pose que des problèmes qu'il est déjà presque impossibie de résoudre. Le temps qui passe n'est pas le signe du manque de consistance du monde sens.ble, par opposition au calme éternel du monde des idées, il n'est pas le faux par opposition au vrai, mais l'espace étroit et évanescent où la vérité peut éclater, mais où aussi elle peut être tue et cachée: il n'y a de vérité que précipitée. Les vérités "éterneles" sont, certes, respectables, mais elles n'ont jamais de rapport direct avec ce qui importe, le salut individuel ou collectif. On commence à comprendre que des connaissances exactes puissent être moins importantes ou utiles que certaines opinions raisonnables. C'est le rapport entre la doxa et l'epistemê qui a été renversé.

$\mathrm{Si}$ ce renvoi à Isocrate est structuralement correct (car il ne s'agit pas évidemment de parler d'"influence", la seule référence de Rousseau à Isocrate étant, à notre connaissance, purement extrinsèque), il peut nous aider à trouver le fil conducteur de la lecture et, avec lui, la clef de l'ordre qui unifie l'oeuvre et lui donne cohérence. L'apparence de désordre et l'illusion de contradiction, dénoncées déjà par Rousseau, pourraient bien être l'effet d'une lecture qui ignore l'organisation rhétorique de l'oeuvre, la manière par laquelle elle s'ordonne à un auditoire particulier. Si le discours de Rousseau est commandé par cette vocation rhétorique, il serait

(74) O. C., I, p. 1011. 
vain de chercher à reconstituer la totalité de son oeuvre dans un espace ou dans un temps homogènes, sans prendre en considération la géographie variable et hétérogène de ses publics. Dans le discours dialectique, le progrès de la connaissance finit par effacer la personnalité des interlocuteurs, que perdent leur identité devant l'éclat de l'universa'ité de la science et deviennent le milieu purement passif de la causalité de l'idée (75); dans le discours rhétorique, au contraire, l'auditeur et le rhéteur gardent leur identité et leur profil concret. Ce discours n'a pas l'intention d'incarner et de dévoiler la ratio immuable, mais de donner, à l'intérieur d'une situation concrète et d'une humanité particulière, um simulacre de l'Ordre. Ce n'est pas la vérité universelle qu'il veut rendre visible, mais une certaine vérité, utile hic et nunc à cette humanité locale. Découvrir l'ordre du discours rhétorique présuppose la réponse à la question suivante: qui parle et à qui? Dans la mesure où le rhéteur se situe par rapport à un auditoire particulier et prend en considération sa "psychologie" et ses préjugés, il acquiert lui-même un profil particulier et son visage ne peut pas être simp'ement le truchement d'une raison universelle. Il doit même, quelquefois, ajuster sur son visage un masque et cacher des vérités, car "La vérité particulière n'est pas toujours un bien, elle est quelquefois un mal, très souvent une chose indifférente" (76).

C'est dans une certaine conception de la vérité que se fonde la consistance propre du discours rhétorique, dans ce que nous pourrions appeler une éthique de la vérité ou une politique de l'entendement. Cette éthique de la vérité s'articule à la charnière de trois notions différentes: vérité universelle, vérité particulière et Justice. Regardons, par exemple, une lettre adressée à d'Alember:

“... Quant à ceux qui trouvent ou feignent de trouver de l'opposition entre ma Lettre sur les spectacles et la Nouvelle Hé.ô̂se, je suis bien sur qu'ils ne vous imposent pas. Vous savez que la vérité, quoiqu'elle soit une, change de forme selon les temps et les lieux, et qu'on peut dire à Paris ce qu'en des jours plus heureux on n'eut pas dû dire à Genève" (77).

Ce texte nous intéresse non seulement dans la mesure où il dit explicitement que l'illusion de la contradiction ne peut s'imposer qu'au lecteur qui ignore à qui est adressé le discours (et dans la mesure où il semble ainsi confirmer explicitement notre hypothèse) mais surtout parce qu'il nous aiguille vers le fondement où la rhétorique puise sa vérité propre et sa légitimité. Ce texte ne vise pas,

(75) V.Goldschmidt, La religion de Platon, Presses Universitaires de France.

(76) O. C., I, p. 1026.

(77) O. C., ed. Furne et Cie, IV, p. 329. 
en effet, à dissoudre la notion de vérité dans un relativisme anarchique et à dire simplement que ce qui est vrai en deçà des Pyrenées ne l'est plus au delà. Ce n'est pas, à proprement parler, la vérité qui change, mais l'opportunité de sa manifestation: c'est le devoir de dire ou de taire une vérité qui varie de Paris à Genève, ou à Genève même, avant ou après l'arrivée de Voltaire. Mais quelle est la ligne de partage qui sépare les vérités qu'on doit ou qu'on peut dire des vérités qu'on doit et qu'on peut légitimement taire? Elle est dessinée par une sorte de contrat qui unit le rhéteur à son auditoire et ce contrat, à son tour, acquiert son fondement dans l'opposition entre véracité et vérité. C'est cette opposition qui déplace la notion de vérité de son champ traditionnel - celui du rapport entre le discours et l'objet du discours ou entre l'entendement solitaire et le monde des étants - pour le situer à l'intérieur du champ de l'intersubjectivité. Il est évident que cette réfraction de la vérité dans les mailles de l'intersubjectivité n'atteint pas la vérité générale que "est toujour un bien" (78); mais elle n'est pas alors de l'ordre du contenu ou des thèses, elle n'est que 1'“oeil de la raison" ou la lumière qui apprend à l'homme à choisir et se conduire. Mais dès que nous passons de cette lumière universelle aux contenus, aux vérités particulières, elles seront, par rapport aux humanités locales, des biens, des maux ou simplement des choses indifférentes. Et le critère qui nous permet de distinguer entre les différentes sortes de vérités est la Justice: la limitation du champ de la vérité, le choix des "bonnes" vérités est fondé dans l'amour de la justice qui précéde et fonde l'amour de la vérité, il est fondé dans un rapport à l'autre dans l'horizon d'une humanité particulière:

"Mais, diroit-on, comment accorder ce relâchement avec cet ardent amour pour la vérité dont je la glorifie? Cet amour est donc faux puisqu'il souffre tant d'alliages? Non, il est pur et vrai: mais il n'est qu'une émanation de l'amour de la justice et ne veut jamais être faux quoiqu'il soit souvent fabuleux. Justice et vérité sont dans son esprit deux mots synonymes qu'il prend l'un pour l'autre indifféremment" (79).

Le sage ne parle donc jamais d'un lieu inassignable - partout et nulle part - auquel il aurait accés par une ascèse intellectuelle, mais toujours d'un lieu particulier, à Genève ou à Paris, auquel il est lié par l'exigence plus fondamentale de la Justice. C'est parce qu'il ignore ce compromis éthique avec l'auditoire particulier, c'est parce qu'il le dissout dans le fond d'un prétendu auditoire universel, que le philosophe est nécessairement méchant. Ignorant le contrat local qui constitue cette humanité particulière à laquelle il s'adresse, 
il contribue à le dissoudre et son discours devient nécessairement injuste: l'ambition d'universalité de la philosophie ne fait que détruire les liens de la sociabilité réelle, sans rendre possible aucune humanité universelle:

"Le goût de la philosophie relâche tous les liens d'estime et de bienveillance qui attachent les hommes à la société, et c'est peut-être le plus dangereux des maux qu'elle engendre" $(80)$.

Rousseau nous avertit donc explicitement que son discours s'adresse à des auditoires particuliers, que son sens et sa vérité ne peuvent pas être saisis sans la conscience de la "situation rhétorique" dans laquelle il a été produit. Mais plus qu'une référence à l'idée d'auditoire particulier, on peut trouver dans ses textes une véritable théorie des auditoires, de leur structure et de leur histoire, une typologie générale des auditoires. Cette théorie est indissociable de sa théorie des langues et de sa théorie de l'Histoire. Nous pouvons trouver une indication de cette imbrication dans le dernier chapitre de l'Essai sur l'origine des langues qui examine le rapport des langues aux gourvenements:

"Les langues se forment naturellement sur les besoins des hommes; elles changent et s'altèrent selon les changemens de ces mêmes besoins. Dans anciens temps, où la persuasion tenait lieu de force publique, l'éloquence était nécessaire. A quoi servirait-elle aujourd'hui, que la force publique supplée à la persuasion? L'on n'a besoin ni d'art ni de figure pour dire, tel est mon plaisir. Quels discours restent donc à faire au peuple assemblé? Des sermons. Et qu'importe à ceux qui les font de persuader le peuple, puisque ce n'est pas lui qui nomme aux bénéfices? Les langues populaires nous sont devenues aussi parfaitement inutiles que l'éloquence. Les sociétés ont pris leur dernière forme: on n'y change plus rien qu'avec du canon et des écus: et comme on n'a plus rien à dire au peuple, sinon, donnez de l'argent, on le dit avec dcs placards au coin des rues, ou des soldats dans les maisons. Il ne faut pas assembler personne pour cela: au contraire, il faut tenir les sujets épars: c'est la première maxime de la politique moderne" (81).

Ce texte qui a trait aux limites de l'éloquence dans le monde moderne (et qui semble ainsi limiter la portée de la rhétorique)

(80) O. C., II, p. 967.
(81) Essai sur l'origine des langues, reproduction off-set de l'édition Dupuis '1817), Cahiers pour l'Analyse, 1967, p. 542. 
montre dans la réalité comment l'idée de l'eloquence est centrale dans la conception que Rousseau se fait et des langues en général et de l'histoire des sociétés. Les limites de l'éloquence sont au fond les limites de la langue elle-même, ainsi que de la liberté et de l'humanité. Mais entre un pô'e et l'autre, les anciens temps où le langage était la seule force et "aujourd'hui" où la force est seul langage, il y a tout un continu et une histoire où les rapports entre langage et violence dessinent une série de figures différentes. Chacune de ces configurations est définie par les rapports entre la langue et le gouvernement, entre le langage et le pouvoir. C'est cette manière d'articuler un gouvernement et une langue, ce degré particulier de pouvoir de langage qui définit un auditoire particulier. Ce qu'il y a de tranchant dans ce texte - qui désigne plus les limites de l'Histoire que son déroulement réel - est nuancé dans d'autres textes qui reconnaissent la spécificité et la différence des genevois, des parisiens, des polonais et des corses. C'est ainsi, par exemple, que dans le très intéressante préface de la Nouvelle Héloîse, la justification du roman passe par l'opposition de deux types de lecteurs; pour répondre aux objections de l'éditeur, il faut passer par la critique de l'idée que le roman doit où peut s'adresser à un public universel:

" $R$. La raison qui vous feroit supprimer cet Ouvrage m'encourage à le publier.

N. Quoi! La certitude de n'être point lu?

$R$. Un peu de patience, et vous allez m'entendre.

En matière de morale, il n'y a point, selon moi, de lecture utile aux gens du monde. Premiéremcnt, parce que la multitude des livres nouveaux qu'is parcourent, et qui disent tour-à-tour le pour et le contre, détruit l'effet de l'un par l'autre, et rend le tout comme non avenu. Les livres choisis qu'on relit ne font point d'effet encore; s'ils soutiennent les maximes du monde, ils sont inutiles; et s'ils les combattent, ils sont inutiles. Ils trouvent ceux qui les lisent liés aux vices de la société, par des chaînes qu'ils ne peuvent rompre. L'homme du monde qui veut remuer un instant son âme pour la remettre dans l'ordre moral, trouvant de toutes parts une résistance invincible, est toujours forcé de garder ou de reprendre sa prémière situation. Je suis persuadé qu'il y a peu de gens bien nés qui n'ayent fait cet essai, du moins une fois en leur vie; mais bientôt découragé d'un vain effort on ne le répète plus, et l'on s'accoutume à regarder la morale des livres comme un babil de gens oisifs. Plus on s'éloigne des affaires, des grandes villes, des nombreuses sociétés, plus les obstacles diminuent. Il est un terme où ces obstacles cessent d'être invincibles, et c'est alors que les livres peuvent avoir quelque utilité. Quand on vit isolé, comme on ne se hâte pas de lire pour faire parade de ses 
lectures on les varie moins, on les médite devantage; et comme elles ne trouvent pas un si grand contrepoids au-dehors, elles font beaucoup plus d'effet au-dedans. L'ennui, ce fléau de la solitude aussi-bien que du grand monde, force de recourir aux livres amusans, seule ressource de qui vit seul et n'en a pas en lui-même. On lit beaucoup plus de romans dans les Provinces qu'à Paris, on en lit plus dans les campagnes que dans les villes, et ils y font beaucoup plus d'impression: vous voyez pourquoi cela doit être" (82).

On pourrait voir de l'ironie dans ce texte, car elle ne manque pas au long de la préface. Mais le sérieux de l'affirmation et l'importance de la thèse est attesté par un passage des Confessions où, flatté par le succès parisien de son roman, il est en même temps embarrassé, car ce succès semble démentir non seulement sont attente, mais aussi une thèse fondamentale de sa pensée. K'est encore l'idée de simulacre qui lui permet alors de résoudre ce problème: "L'amitié, l'amour, la vertu régnent-ils donc à Paris plus qu'ailleurs? Non sans doute; mais il y régne encore ce sens exquis qui tronsporte le coeur à leur image, et qui nous fait chérir dans les autres les sentimens purs, tendres, honnêtes que nous n'avons plus" (83).

L'importance de l'idée d'auditoire particulier est visible aussi dans les conséquences qu'elle déclenche dans le domaine de la conception des genres littéraires. La reconnaissance du caractère local de tout discours détruit l'idée que les différents genres correspondent à des essences immuables et fixées dans un ciel quelconque: les genres doivent être compris sur le fond d'une historicité qui libère leur possibilité ou qui les rend non viables et les expulse vers le monde extérieur du non-sens. C'est ce que nous pouvons voir en comparant les textes où Rousseau parle de l'éloquence politique et du roman. Il s'agit moins de deux genres différents: ce sont plutòt les formes de discours que sont possibles au citoyen dans des situations historiques différentes. Le rapport établi entre le pouvoir et le langage dans la Cité antique libérait la possibilité de l'éloquence politique, celui qui gouverne la société moderne n'ouvre que l'étroite et misérable porte du roman. Tel est le sens qu'il faut donner à la phrase de St. Preux: 
"Les Romans sont peu-être la dernière instruction qu'il reste à un peuple assès corrompu pour que toute autre lui soit inutile" (84).

Il est, en effet, curieux de noter que dans la préface de la Nouvelle Hélö̈se, ansi que dans le dernier chapitre de l'Essai sur l'origine des langues, l'éloquence politique et le Roman sont définis d'après le même procédé, c'est-à-dire, dans leur commune opposition aux sermons. Dans les deux cas, le sermon est défini comme discours impuissant, incapable de mouvoir son auditoire:

"Il y a des langues favorable à la liberté; ce sont les langues sonores, prosodiques, harmonieuses, dont on distingue le discours de fort loin. Les nôtres sont faites pour le bourdonnement des divans. Nos prédicateurs se tourmentent, se mettent en sueur dans les temples, sans qu'on sache rien de ce qu'ils on dit. Après s'être épuisés à crier pendant une heure, ils sortent de la chaire à demi morts. Assurement ce n'était pas la peine de perdre tant de fatigue” (85).

Ce sont bien nos langues qui font du sermon un discours inutile, mais le manque de force et persuasion de la langue renvoie à la forme de gouvernement et à un manque de force de l'âme elle-même: même si le Prédicateur était écouté, il ne serait pas suivi. Car le propre du Prédicateur est son style pour ainsi dire normatif: il propose la vertu comme un devoir à des gens qui sont incapables de se plier à un devoir. Mais ce que le Prédicateur est incapable de faire, devient possible au romancier, s'il est un vrai citoyen. On a pu dire que la Nouvelle Héloüse était un "rêve de volupté redressé en instruction morale": mais il n'y a rien de paradoxal dans ce passage du rêve de volupté à l'instruction morale, car sans flatter la volupté d'un pubiic structurellement volupteux toute instruction morale n'est qu'un voeu pieux; c'est Rousseau qui le dit, en définissant le bon usage possible des romans:

"C'est le métier des Prédicateurs de nous crier: Soyez bons et sages, sans beaucoup s'inquietter du succès de leurs discours; le citoyen qui s'en inquiette ne doit point nous crier sotement: soyez bons; mais nous faire aimer l'état qui nous porte à l'être" (86).

Dernier ersatz de l'éloquence civique des anciens, le roman ne s'oppose pas au discours politique et la littérature ne peut pas être pensée comme l'autre de la théorie politique.

(84) O. C., II, p.

(58) Essai sur l'origine des langues, p. 542. 
Telles sont les considérations qui nous on conduit à formuler les hypothèses suivantes, dont la démonstration est le but de notre travail:

1) Il y a chez Rousseau une théorie génerale de la rhétorique, des conditions concrètes (psychologiques, sociales, linguistiques) de la persuasion.

2) Cette rhétorique domine tous les genres littéraires - oeuvres de raisonnement et oeuvres d'agrément - et les organise dans la continuité d'un champ homogène.

3) L'oeuvre de Rousseau elle-même est commandée par les principes de la rhétorique qu'il propose et son apparente excentricité manque d'unité ou de cohérence - disparait si la lecture se fait selon une centration rhétorique. L'unité de la pensée de Rousseau peut être montrée dans le mouvement même de ses écrits, c'est-à-dire, selon la stratégie de la persuasion ou selon l'ordre de l'argumentation.

Proposer ces trois hypothèses était le but de cette introduction . Avant de mettre un point final à ces considérations introductoires il est utile, toutefois, d'anticiper au moins une objection qui n'aura certainement pas échappé au lecteur. Comment, en effet, faire de la rhétorique le point privilégié de la lecture de l'auteur des Rêveries? Comment faire de la référence à un auditoire particulier quelque chose d'essentiel pour quelq'un qui ouvre un livre, chaque fois plus reconnu comme central, avec les mots suivants:

"Me voici donc seul sur la terre, n'ayant plus de frère, de prochain, d'ami, de société que moi-même. Le plus sociable et le plus aimant des humains en a été proscrit par un accord unanime. Ils ont cherché dans les rafinemens de leur haine quel tourment pouvoit être le plus cruel à mon âme sensible et ils ont brisé violemment tous les liens qui m'attachaient à eux. Ils n'ont pu qu'en cessant de l'être se dérober à mon affection. Les vôlà donc étrangers, inconnus, nuls enfin pour moi puis qu'ils l'ont voulu. Mais moi, détaché d'eux et de tout, que suis-je moi-même? Voila ce qui me reste à chercher" (87).

Où est l'intention rhétorique dans ce discours qui semb'e être coupé de tout auditoire et que renonce à toute persuasion possible en

O. C., II, p. 20.

O. C., I, p. 995 . 
s'excluant de l'humanité? De quel auditoire peut on parler devant une telle voix te'lement tournée vers soi et fermée à l'écoute d'elle-même, que le lecteur peut se sentir comme un intrus? La réponse facile - et qu'il faut exclure — ce serait d'exclure aussi ce discours extrême de l'intérieur de l'oeuvre, de voir en lui quelque chose comme un au-delà de l'oeuvre qui ne commence que quand elle abandonne la théorie vers quelque chose qui la dépasse. On pourrait même prendre Rousseau sur paro'e et rappeler le texte où il semble, lui-même, banir de "l'oeuvre" des écrits comme la Lettre à Chr. de Beaumont et les Lettres écrites de la Montagne car il s'agirait de documents qui ne seraient "destinés qu'à la défense personnelle de sa patrie et de son honneur" (88). Mais l'évident intérêt philosophique des Rêveries nous interdit cette facilité: son exclusion ne serait que l'aveu de la limitation de la lecture proposée.

Est-ce qu'il faudrait donc revenir en arrière et biffer les propositions avancés? Il semble, en effet, qu'un écrit comme les Rêveries n'est justiciable que d'une interprétation qui vise moins l'ordre du discours que l'expérience qui le précède et qu'il exprime. La rhétorique de Rousseau ne serait que l'expression inessentielle d'une expérience fondamentale qui seule peut l'exp'iquer. Commentant le premier Discours, J. Starobinski fait l'observation suivante: "Cette rhétorique sert de véhicule à une pensée obsedée par l'idée de l'impossibilité de la communication humaine" (89). Cette perspective non seulement expliquerait les Rêveries comme point final d'un itinéraire en meme temps intellectuel et existentiel, mais aussi toutes ses étapes antérieures. Car toujours, comme nous l'avons déjà vu, Rousseau insiste sur les limites de la persuasion et sur l'inefficacité qui menace toujours la rhétorique. C'est bien une rhétorique désespérée celle qui touche à sa propre limite et qui découvre qu'elle ne peut persuader qu'à celui qui lui a déjà accordé son assentiment intérieur. C'est le désespoir qui fait l'étrangeté des Dialogues où on peut aussi lire:

"Je m'expliquerai: mais ce sera prendre le soin le plus inutile ou le plus superflu: car tout ce que je vois ne saurait être entendu que par ceux à qui l'on n'a pas besoin de le dire" $(90)$.

La rupture totale qui est à l'origine des Rêveries et que Rousseau présente comme le dernier terme de son itinéraire était déjà inscrite comme possibilité tout au long du passé. Quel est donc ce destin que Rousseau décrit souvent sous la forme d'un enchaînement nécessaire? C'est la phrase "J'ai pris le parti d'écrire et

(88) O. C., I, p. 933 (cf. la note 3).

(89) J. Starobinski, Jean-Jacques Rousseau, La Transparence et l'Obstacle, p. 3.

(90) O. C., I, p. 668. 
de me cacher", si bien commentée par J. Staronsbiski, qui fournirait la clef de cet itinéraire. Au point de départ, il y a la volonté ou le désir de la transparence; mais dès le point de départ il y a, l'expérience de la violence, de l'obstacle, de la transparence comme le paradis à jamais perdu. L'écriture apparait alors comme le biais qui pourrait permettre de reconquérir ce paradis: écrire c'est renoncer à la communication immédiate, mais écrire c'est aussi préparer le retour à l'immédiat. En écrivant, Jean-Jacques se cache sous le masque de l'Auteur, mais li attend le moment où ce masque deviendra superflu et où l'oeuvre s'effacera pour laisser place à la communauté des coeurs transparents. Mais c'est le "complot" et sa généralisation universelle ("... par un accord unanime") qui viennent déjouer la stratégie de l'auteur et les ruses du désir. Coupant ainsi cruellement ("... ils ont brisé violemment...") le projet le plus profond de Jean-Jacques, le complot devrait nécéssairement changer son rapport à l'écriture: le supplément inessentiel de la présence devient le seul espace où elle peut se survivre et être préservée dans son propre simulacre. Étrange projet, en effet, pour un contempteur des livres et de l'écriture que d'essayer de garder quelques pages écrites à l'intérieur de l'espace sacré d'un Autel. C'est comme si l'anticipation de la mort - dans la certitude qu'il ne reste aucun témoin de son innocence - eût donné une autre consistance à l'oeuvre, devenue seul garant d'une présence qui se voit condamnée au néant. Le lecteur futur, lecteur improbable mais seul lecteur possible, est désormais le seul espoir de faire renaître un jour la certitude d'une innocence qui n'avait d'elle-même qu'une incertaine certitude.

Mais alors cette découverte pathétique des limites de la rhétorique pourrait être comprise comme étant déjà le signe de la naissance de la littérature. C'est d'ailleurs ce que dit Maurice Blanchot: la solitude de Rousseau n'est que l'autre côté, l'effet pour ainsi dire existentiel, de la découverte d'un nouveau langage - parole solitaire - du langage de la littérature:

"J'ai toujours soupçonné ce vice profond et insaisissable (le "vice" qui selon Blanchot, est à l'origine de la difficulté du commentaire de Rousseau) d'être celui auquel nous devons la littérature. Rousseau, l'homme du commencement, de la nature et de la vérité, est celui qui ne peut accomplir ses rapports qu'en écrivant; écrivant, il ne peut que les faire dévier de la certitude qu'il en a: dans cette déviation dont il souffre, qu'il récuse avec élan, avec désespoir, il aide la littérature à prendre conscience d'elle-même en se dégageant des conventions anciennes et à se former, dans la contestation et les contradictions, une rectitude nouvelle" (91).

(91) M. Blanchot, op. cit., pp. 53-A. 
$\mathrm{Si}$ le langage de Rousseau a bien cette nature, il faut dire, en effet, qu'il est l'autre de la théorie ou, comme le dirait Blanchot, qu'il est absolument extérieur à tout Savoir; ce langage, Blanchot nous le décrit comme coupé de toute référence aux objets - sa vérité n'est pas celle du monde. Plus encore, ce langage ne serait jamais adressé à un interlocuteur, il surgirait de sa propre impossibilité et ne renvoierait qu'à un lecteur "infiniment futur".

"Dans la mesure où écrire, c'est s'arracher à l'impossibilité, où écrire devient possible, écrire assume alors les caractères de l'exigence de lire, et l'écriture devient l'intimité naissante du lecteur encore infiniment futur" (92).

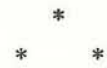

A ces objections, il faut opposer les considérations suivantes: ce qu'on a appelé le "désespoir" de la rhétorique ne correspond pas nécessairement à la naissance de la littérature, telle que la définit $M$. Blanchot. En effet, le lecteur infiniment futur dont parle Blanchot est aussi paradoxalement contemporain au texte écrit, il est même produit par ce texte, comme un développement d'une "exigence de lecture" qui lui est interne. C'est ainsi que le texte ci-dessus est précedé et expliqué par la description de la genèse du "lecteur" comme un processus immanent à l'oeuvre elle-mềme:

"L'on dit quelquefois que tout auteur écrit en présence de quelque lecteur ou encore pour être lu. C'est une manière de parler peu réfléchie. Ce qu'il faut dire, c'est que la part du lecteur, ou de ce qui deviendra, une fois l'oeuvre faite, pouvoir ou possibilité de lire, est déjà présente, sous formes changeantes, dans la genèse même de l'oeuvre" (93).

Il s'agit d'une manière "peu réfléchie" de parler, en effet, quand il s'agit de la littérature, de ce que sont devenues les Belle Lettres dans l'horizon de la modernité, par une crise qui met en question leur essence et leur fondement et qui fait de la question du fondement des Lettres le sens même des Lettres:

"Qu'est-ce que l'oeuvre? Qu'est-ce que le langage dans l'oeuvre? Quand Mallarmé se demande: "Quelque chose comme les Lettres existe-t-il?", cette question est la littérature même, elle est la littérature quand celle-ci est devenue le souci de sa propre essence. Une telle question ne peut être reléguée. Qu'arrive-t-il par le fait que

(92) M. Blanchot, L'espace littéraire, "Idées", Gallimard, p. 267.

(93) Ibid. 
nous avons la littérature? Qu'en est-il de l'être, si l'on dit que "quelque chose comme les Lettres existe"?" (94).

Il s'agit d'une manière "peu réfléchie" de parler encore et sourtout parce que, dans cette métamorphose, le lecteur et l'auteur perdent autonomie devant l'oeuvre, devenue quelque chose comme un langage absolu. Qu'est-ce que le lecteur ou l'auteur, en effet, quand on vérifie que "désormais, ce n'est pas Mallarmé qui par'e, mais le langage se parle, le langage comme oeuvre et l'oeuvre du langage" (95).

Chez Rousseau, au contraire, c'est bien Jean-Jacques qui parle et le lecteur futur est simplement une probabilité et s'il peut un jour devenir réel, il le deviendra à l'intérieur d'une génération déterminée, dans un Siècle déterminé: le "lecteur futur" de Rousseau n'est pas cette ombre que l'oeuvre, dans son autarcie, projete au delà d'el'e-même.

De même, il faut reconaître qu'il n'y a pas de rhétorique qui ne connaisse ses propres limites, qui ne se produise justement à la limite de ses possibilités. $\mathrm{Si}$, en effet, la rhétorique nait de la reconnaissance de l'impossibilité de vaincre ou de convaincre, elle est toujours à la merci de la résistance de l'auditoire: elle essaye de persuader en s'arrachant à l'impossibilité de convaincre. Toute rhétorique, pourrait-on dire (dans la mesure où elle se constitue à partir de la résistance de son auditoire et de la certitude que l'assentiment ne peut pas être arraché) est déjà une rhétorique désespérée. Pour mieux préciser les termes de la question - et en particulier celui du lieu des Rêveries - il est nécessaire de revenir sur les rapports entre le rhéteur et son auditoire. Il ne s'agit pas, en effet, d'un rapport simple et il ne peut pas être compris par le simple renvoi à un auditoire particulier.

Quelle est la tâche du rhéteur selon Rousseau, et quel lieu doit-il occuper par rapport à son auditoire? Le personnage du rhéteur ne peut pas être compris sans la référence à deux autres personnages essentiels de la pensée de Rousseau et dont il ne peut pas à la limite être distingué: le législateur et l'ethnologue. Mais sous quel rapport peuvent le législateur et l'ethnologue être considérés comme simétriques? Les deux personnages sont essentiellement des étrangers, ils viennent du dehors vers une humanité particulière. On ne peut ni connaître ni instituer que l'Autre. L'importance epistémologique de l'altérité et de la différence a été particulièrement soulignée par Lévi-Strauss dans son essai sur Rousseau. De

(94) Ibid., p. 40.

(95) Ibid., p. 38. 
même, ces éléments seront aussi essentiels dans l'instituition d'un peuple:

\begin{abstract}
"Quand Lycurgue donna des loix à sa patrie, il commença par abdiquer la Royauté. C'était la coûtume de la plupart des villes grecques de confier à des étrangers l'établissement des leurs. Les Républiques modernes de l'Italie imiterent souvent cet usage: celle de Genève en fit autant et s'en trouva bien. Rome dans son plus bel âge vit rennaitre en son sein tous les crimes de la Tirannie, et se vit prête à périr, pour avoir réuni sur les mêmes têtes l'autorité législative et le pouvoir souverain" (96).
\end{abstract}

Seul l'étranger, qui ne partage pas les préjugés et les intérêts de cette humanité locale, peut s'approcher de cette condition extraordinaire qui est celle du législateur. Son "emploi" même est extérieur à l'instituition qu'il rend possib'e: "Ce n'est point magistrature, ce n'est point souveraineté. Cet emploi, qui constitue la république, n'entre pas dans sa constitution ...” (97). Ce minimum d'extériorité est la condition de l'apparition de la Raison au sein de la Cité - comme l'extériorité du nous poietikos était condition de la rationalité de l'âme chez Aristote.

Le rhéteur est celui qui, "connaissant l'homme", s'adresse aux hommes, qui parie à une humanité particulière de cet espace vide qui sépare les humanités particulières. Il est l'homme de la nature justement parce qu'il n'habite entièrement aucune cité, parce qu'il habite l'espace qui les sépare. Voilà le fondement de son exemplarité, de ce qui donne à la contingence de son destin une portée universelle. Il ne peut donc pas adhérer complètement à son auditoire particulier, il est séparé de lui par l'abîme de la différence, sans laquelle son discours n'aurait pas d'intérêt: sans cette différence, le discours n'aurait d'autre fonction que de confirmer l'auditoire particulier dans ses préjugés et n'aurait aucun pouvoir édifiant.

Mais si la rhétorique est bien ainsi le discours de l'Autre, elle est toujours hantée par la possibilité de devenir un discours solitaire. Les Rêveries ne contredisent pas l'idée que toute l'oeuvre de Rousseau est centrée sur un projerhétorique, elles réalisent une possibilité qui est inscrite dans la structure ironique du discours rhétorique. L'ironie de la dialectique est plus lourde que celle de la rhétorique: devant l'interlocuteur qui résiste, le dialecticien doit le vaincre, car réfuter la thèse de Trasimaque c'est réfuter Trasimaque. L'ironie de la rhétorique est p'us souple car elle peut

(96) O. C., III, p. 382.

(97) Ibid. 
même feindre qu'elle ignore son interlocuteur et cacher que son silence même est un argument. Nous avions mis en exergue de cette dernière partie de notre introduction une phrase où Rousseau fait l'éloge de l'éloquence des anciens: il est venu le moment de la compléter par les exemples qui l'il'ustrent et qui font penser à une rhétorique de l'exemple et de l'ilustration.

"Trastibule et Tarquin coupant des têtes de pavots, Alexandre appliquant son sceau sur la bouche de son favori, Diogène marchant devant Zenon ne parlaient-ils pas mieux que s'ils avoient fait de longs discours?" (98).

Ce texte veut évidemment insister sur l'éloquence des signes premiers, il veut montrer dans ce langage immédiat quelque chose d'infiniment plus persuasif que les discours les plus articulés. Mais ou peut y voir aussi une sorte de théorie de l'exemple. Rousseau, abandonnant le monde, refusant de s'adresser à n'importe qui, n'estil pas aussi une sorte de Diogène qui se refuse à parler et qui commence à marcher devant Zenon? Parler, ce serait jouer le peu qu'on veut montrer comme fallacieux. En effet, au rhéteur qui a apparemment épuisé son discours, il reste toujours cette ressource extraordinaire: son existence, même si elle s'enferme dans la plus radicale solitude, sera toujours comme une "figure" ou un "exemple" proposé aux autres:

"Je forme une entreprise qui n'eut jamais d'exemple, et dont l'exécution n'aura point d'imitateur. Je veux montrer à mes semblables un homme dans toute la vérité de la nature; et cet homme ce sera moi" (99).

Que peut, en effet, être un exemple sans imitateur? L'idée même d'exemple est indissociable de quelque chose comme une imitation possible, elle imp'ique nécessairement un minimum de normativité, et la norme renvoie toujours à ce qui doit être normalisé. Un exemple sans imitation possible ne peut être compris que comme une sorte de défi — c'est-à-dire comme une exhortation . C'est l'ironie de la rhétorique qui lui permet ainsi de s'adresser à son auditoire, de lui proposer des exemples sous l'apparence du refus.

(98)

O. C., IV, p. 647 .

O. C., I, p. 5 . 
L'itinéraire de Rousseau - dont Starobinski fournit l'intellig.bilité existentielle - pourrait être ainsi compris aussi par le schéma de la théorie de l'argumentation. Il faudrait simplement superposer à la triade de l'expérience vécue - désir, objet du désir et l'oeil normatif - la triade donnée par la structure ironique de la rhétorique: le rhéteur, son auditoire particulier et l'Autre de cet auditoire, c'est-à-dire, le témoin du rhéteur: ce lecteur futur toujours possible.

BENTO PRADO JR. 